\title{
Gluon-initiated production of a Kaluza-Klein gluon in a bulk Randall-Sundrum model
}

\author{
Benjamin C. Allanach, ${ }^{a}$ Farvah Mahmoudi, ${ }^{b}$ Jordan P. Skittrall ${ }^{a}$ and K. Sridhar ${ }^{c, d}$ \\ ${ }^{a}$ Department of Applied Mathematics and Theoretical Physics, \\ Centre for Mathematical Sciences, Univeristy of Cambridge, \\ Wilberforce Road, Cambridge, CB3 0WA, U.K. \\ ${ }^{b}$ Laboratoire de Physique Corpusculaire de Clermont-Ferrand (LPC), \\ Université Blaise Pascal, CNRS/IN2P3, \\ 63177 Aubière Cedex, France \\ ${ }^{c}$ LAPTH, Univ. de Savoie, CNRS, \\ BP-110, F-74941, Annecy-le-Vieux, France \\ ${ }^{d}$ Department of Theoretical Physics, Institute of Fundamental Research, \\ Homi Bhabha Road, Bombay 400005, India \\ E-mail: B.C.Allanach@damtp.cam.ac.uk, mahmoudi@in2p3.fr, \\ J.P.Skittrall@damtp.cam.ac.uk, sridhar@theory.tifr.res.in
}

Abstract: In the Bulk Randall-Sundrum model, the Kaluza-Klein excitations of the gauge bosons are the primary signatures. In particular, the search for the Kaluza-Klein (KK) excitation of the gluon at hadron colliders is of great importance in testing this model. At the leading order in QCD, the production of this KK-gluon proceeds only via $q \bar{q}$-initial states. We study the production of KK-gluons from gluon initial states at next-to-leading order in QCD. We find that, even after including the sub-dominant KK-gluon loops at this order, the next-to-leading order (NLO) cross-section is tiny compared to the leading order cross-section and unlikely to impact the searches for this resonance at hardon colliders.

Keywords: Field Theories in Higher Dimensions, Beyond Standard Model, NLO Computations 


\section{Contents}

1 Introduction $\quad 1$

1.1 Theoretical strategy 3

1.2 Notation 4

2 General form of the amplitude $\quad 4$

$\begin{array}{lll}3 & \text { Feynman rules applicable to the calculation } & 6\end{array}$

$\begin{array}{lll}4 & \text { Diagrams } & 10\end{array}$

$\begin{array}{lll}4.1 & \text { Quark loop diagrams } & 10\end{array}$

$\begin{array}{lll}4.2 & \text { Kaluza-Klein gluon (and ghost) loop diagrams } & 12\end{array}$

$\begin{array}{lll}4.3 & \text { Counterterm diagrams } & 15\end{array}$

5 Calculation of the amplitude $\quad 16$

$\begin{array}{ll}5.1 \text { Simplification of the calculation } & 16\end{array}$

$\begin{array}{lll}5.2 & \text { Contribution from diagrams with quark loops } & 17\end{array}$

5.3 Contribution from diagrams with Kaluza-Klein gluon (or ghost) loops 19

$\begin{array}{ll}5.4 & \text { Overall amplitude before anomaly cancellation } \\ 5.5 & 20\end{array}$

$\begin{array}{ll}5.5 & \text { Cancellation of the anomaly } 20\end{array}$

6 Calculation of the production cross-section 22

$\begin{array}{lll}7 & \text { Discussion } & 23\end{array}$

A Tensors satisfying the general form of section $2 \quad 25$

B Analytic evaluation of the Feynman parameter integral used in the cal$\begin{array}{lr}\text { culation } & \mathbf{2 6}\end{array}$

C Generalisation of our results to off-shell KK gluon production $\quad 27$

\section{Introduction}

The Randall-Sundrum (RS) model [1] is a 5-dimensional model with the fifth dimension, a slice of anti-de Sitter spacetime with strong curvature, compactified (to a size comparable to the Planck length) on a $\mathbf{S}^{1} / \mathbb{Z}_{2}$ orbifold. Two branes are located at the orbifold fixed points $\phi=0, \pi$, the Planck brane and the TeV brane, respectively. The Standard Model fields are localised on the $\mathrm{TeV}$ brane while gravitons exist in the full five-dimensional spacetime. The five-dimensional spacetime metric is of the form

$$
d s^{2}=e^{-k R_{c} \phi} \eta_{\mu \nu} d x^{\mu} d x^{\nu}+R_{c}^{2} d \phi^{2}
$$


$k$ is a mass scale related to the curvature; $\exp \left(-k R_{c} \phi\right)$ is the warp factor which rescales masses of fields localised on the TeV-brane. The electroweak hierarchy $\frac{M_{P}}{M_{\mathrm{EW}}} \sim 10^{15}$ can be generated by an exponent of order 30 and thus the model provides a solution to the hierarchy problem. For this to work, the compactification radius $R_{c}$ should be stabilised against quantum fluctuations. This can be done by introducing a bulk scalar field which generates a potential that allows for the stabilisation $[2,3]$. The RS model predicts a discrete spectrum of Kaluza-Klein (KK) excitations of the graviton and these couple to the Standard Model fields with a coupling that is enhanced by the warp factor to be of the order of electroweak strength. Several collider implications of these graviton resonances have been studied in the literature [4-7].

Since the original RS model is a model of gravity in AdS spacetime it is possible to relate it using the AdS/CFT correspondence [8] to a dual theory - a strongly coupled gauge theory in four dimensions $[9,10]$. In this description, it turns out that the fields localised on the $\mathrm{TeV}$ brane are $\mathrm{TeV}$-scale composites of the strongly interacting theory making the RS model dual to a composite SM. Such a composite theory is unviable: the simplest possibility is to modify the model so that only the Higgs field is localised on the $\mathrm{TeV}$ brane while the rest of the SM fields are in the bulk [11, 12].

In constructing such variants of the RS model, it is not an easy task to avoid the constraints coming from flavour hierarchy, electroweak precision tests and flavour-changing neutral currents [13-18]. In particular, in order to avoid an unacceptably large contribution to the electroweak $T$ parameter an enhanced symmetry in the bulk like $\mathrm{SU}(2)_{L} \times \mathrm{SU}(2)_{R} \times$ $\mathrm{U}(1)_{(B-L)}$ may be required. The heavier fermions need to be closer to the $\mathrm{TeV}$ brane so as to acquire a large Yukawa coupling through a larger overlap with the Higgs wavefunction. In other words, the profiles of the heavier fermions need to be peaked closer to the TeVbrane. Conversely, the fermions close to the Planck brane will have small Yukawa couplings. However, while the large Yukawa of the top demands proximity to the TeV brane, the lefthanded electroweak doublet, $(t, b)_{L}$, cannot be close to the $\mathrm{TeV}$ brane because that induces non-universal couplings of the $b_{L}$ to the $Z$. Such couplings are strongly constrained by $R_{b}$, the measured branching ratio of $Z \rightarrow b \bar{b}$. So the doublet needs to be as far away from the $\mathrm{TeV}$ brane as allowed by $R_{b}$, whereas the $t_{R}$ needs to be localised close to the $\mathrm{TeV}$ brane to account for the large Yukawa of the top. Even with this choice of profiles the bounds on the masses of the KK gauge bosons, coming from $Z \rightarrow b \bar{b}$ are found to be in the region of $5 \mathrm{TeV}$. A custodial symmetry can be invoked to relax this constraint and it also allows other choices of profiles for the $t_{R}$ and $(t, b)_{L}$. With this custodial symmetry and for appropriate choices of the profiles for the $t_{R}$ and $(t, b)_{L}$ it is found that gauge boson masses as low as $2-3 \mathrm{TeV}$ can be consistent with the constraint from $Z \rightarrow b \bar{b}$ [14-18]. A review of the literature on this subject can be found in reference [19].

The $t_{R}$ localised close to the $\mathrm{TeV}$ brane has an enhanced coupling to the first $\mathrm{KK}$ excitation of the gluon in the bulk and as a result we expect that $g_{\mathrm{KK}} \rightarrow t \bar{t}$ (where $g_{\mathrm{KK}}$ represents the first KK excitation of the gluon) will be a significant decay mode from a discovery perspective. The decay also lends itself to identification via spin determination, since the enhanced coupling to $t_{R}$ over $t_{L}$ means the top quarks from KK gluon decays will be polarised [20]. There will be additional challenges for identification of the $t \bar{t}$ pairs because they 
will be highly boosted in the lab frame, but this channel remains a promising search channel.

From a hadron collider perspective, we are interested in the production process $p p \rightarrow$ $g_{\mathrm{KK}}$. The subprocess $q \bar{q} \rightarrow g_{\mathrm{KK}}$ has already been investigated in some detail for the LHC [20, 21], as well as for the Tevatron [22], but because in many models the light quarks have a relatively suppressed coupling to the $g_{\mathrm{KK}}$, it is worth also considering the process $g g \rightarrow g_{\mathrm{KK}}$, even though this process is one-loop at leading order. It is this process that we consider in this paper. (Other, tree-level, processes, involving $g g$ fusion to $g_{\mathrm{KK}}$ in association with additional top quark production, have also been considered previously [23]. A preliminary study of $g g \rightarrow g_{\mathrm{KK}}$ has already been performed [24], from which our analysis differs by the consideration of an additional channel.)

It might appear prima facie that an extension of Yang's theorem [25] should forbid the on-shell production of a KK gluon from two on-shell Standard Model gluons. However, to apply an extension of the theorem to deduce that an amplitude involving three spin-one particles is zero would require the following conditions to be met:

- The three particles must be on-shell;

- Two of the particles must be massless;

- The amplitude must be symmetric under interchange of the massless particles.

The final condition is not met in the case of our process $g g \rightarrow g_{\mathrm{KK}}$, because the $\mathrm{SU}(3)$ structure of the problem means that the amplitude can contain terms that are antisymmetric under interchange of the Standard Model gluons. More explicitly, an amplitude involving three coloured gauge particles can always be decomposed into two parts: one proportional to $f_{a b c}$ and the other proportional to $d_{a b c}$. Since the $d_{a b c}$ 's are symmetric this part of the amplitude goes to zero due to the usual Yang's theorem arguments. The antisymmetry of the $f_{a b c}$ means that this part of the amplitude picks up an extra sign and survives the restrictions of the Yang's theorem. Therefore, Yang's theorem does not forbid the process $g g \rightarrow g_{\mathrm{KK}}$.

\subsection{Theoretical strategy}

In section 2, an argument regarding the general form that must be taken by the amplitude, similar to those of references [26, 27], shows that it is then sufficient to consider a small subset of possible diagrams in order to derive the overall amplitude. Consideration of the Feynman rules in section 3 will show that our one-loop process can obtain contributions from a large number of diagrams, with loops from fermions, KK excitations of fermions, and KK excitations of gluons, as well as in principle requiring renormalization. In section 4 , we show that it is possible to evade renormalization by considering a particular subset of diagrams, suggested by the general form argument. KK excitations of gluons above the first will be mass-suppressed in the loop, and we therefore neglect diagrams containing such excitations.

In addition, it is in principle possible to add a Chern-Simons term to the Lagrangian of the five-dimensional theory. This term is gauge-dependent, and a particular fivedimensional gauge choice is required to ensure anomaly cancellation in the four-dimensional 
effective theory in which we are working. We start by considering a gauge that is simpler for the loop calculation, and show a posteriori in section 5 that the change of gauge required for anomaly cancellation does not alter any of the diagrams already considered, and produces only one more diagram (containing a scalar line). This additional diagram does not alter the square of the on-shell matrix element. We finally estimate the cross-section for on-shell KK gluon production in section 6, before concluding in section 7 . Technical information is relegated to appendices: in appendix A, we list the tensors satisfying the general form argument, whereas in appendix B, we evaluate a Feynman parameter integral analytically. We provide a recipe for how our results may be adapted to calculate the off-shell KK gluon amplitude in appendix C.

\subsection{Notation}

We shall define the incoming gluon momenta to be $p$ and $q$, with corresponding polarisation tensors $\varepsilon^{\mu}(p)$ and $\varepsilon^{\nu}(q)$ respectively. The outgoing KK gluon momentum is $r$, with corresponding polarisation tensor $\varepsilon_{g_{\mathrm{KK}}}^{\rho}(r)$. The polarisation tensors satisfy

$$
\begin{aligned}
\varepsilon^{\mu}(p) p_{\mu} & =0, \\
\varepsilon^{\nu}(q) q_{\nu} & =0 \\
\varepsilon_{g_{\mathrm{KK}}}^{\rho}(r) r_{\rho} & =0 .
\end{aligned}
$$

The momenta satisfy the on-shell conditions

$$
\begin{aligned}
p^{2}=q^{2} & =0, \\
r^{2} & =M_{\mathrm{KK}}^{2},
\end{aligned}
$$

where $M_{\mathrm{KK}}$ is the mass of the first Kaluza-Klein excitation of the gluon, given approximately by the solution of $[11]$

$$
J_{0}\left(\frac{M_{\mathrm{KK}}}{k} e^{k R_{c} \pi}\right)=0
$$

with $J_{0}$ the Bessel function of the first kind of order zero, $R_{c}$ the radius of compactification of the extra dimension, and $k$ the fixed parameter in the warp factor of order the Planck scale. Four-momentum conservation $(r=p+q)$ yields the on-shell $g_{\mathrm{KK}}$ identity

$$
2 p \cdot q=M_{\mathrm{KK}}^{2} .
$$

Finally, we factor out the polarisation vectors to define the tensor $F_{\mu \nu \rho}(p, q)$ in terms of the matrix element $\mathcal{M}(p, q)$ :

$$
\mathcal{M}(p, q)=\varepsilon_{g_{\mathrm{KK}}}^{\rho *}(r) \varepsilon^{\mu}(p) \varepsilon^{\nu}(q) F_{\mu \nu \rho}(p, q)
$$

\section{General form of the amplitude}

We may simplify the calculation by deriving a general form that must be taken by the amplitude we are calculating. 
QCD current conservation, implied by gauge invariance, results in the properties

$$
\begin{aligned}
p^{\mu} F_{\mu \nu \rho} & =0, \\
q^{\nu} F_{\mu \nu \rho} & =0 .
\end{aligned}
$$

Since there are no tree-level diagrams, we may express $F_{\mu \nu \rho}$ in terms of an expansion in $p$ and $q$. (For more details on the conditions for validity of such an expansion - which are met here - see reference [26].) Expanding first in $p$, we may write

$$
F_{\mu \nu \rho}=\mathcal{T}_{\mu \nu \rho}^{0}(q)+p^{\alpha} \mathcal{T}_{\mu \nu \rho \alpha}^{1}(p, q) .
$$

(We note that this is still a completely general expansion without truncation, because $\mathcal{T}_{\mu \nu \rho}^{0}$ and $\mathcal{T}_{\mu \nu \rho \alpha}^{1}$ are functions of $q$ and of $p$ and $q$, respectively.) Since equation (2.1) must be satisfied for all $p$ with $\left|p_{0}\right| \leq M_{\mathrm{KK}}$ in the centre of mass frame and $p^{2}=0$ (i.e. all physical $p$ ), we can deduce that

$$
\mathcal{T}_{\mu \nu \rho}^{0}=0
$$

and

$$
\mathcal{T}_{\mu \nu \rho \alpha}^{1}=-\mathcal{T}_{\alpha \nu \rho \mu}^{1} .
$$

We may therefore write the amplitude as

$$
\mathcal{M}=\varepsilon_{g_{\mathrm{KK}}}^{\rho *}(r) \varepsilon^{\nu}(q)\left(\varepsilon^{\mu}(p) p^{\alpha}-\varepsilon^{\alpha}(p) p^{\mu}\right) \mathcal{T}_{\mu \nu \rho \alpha}^{1} .
$$

We next expand in $q$, writing

$$
\mathcal{T}_{\mu \nu \rho \alpha}^{1}=t_{\mu \nu \rho \alpha}^{0}(p)+q^{\beta} t_{\mu \nu \rho \alpha \beta}^{1}(p, q) .
$$

Similarly to the previous expansion, we note that since equation (2.2) must be satisfied for all $q$ with $\left|q_{0}\right| \leq M_{\mathrm{KK}}$ in the centre of mass frame and $q^{2}=0$, we can deduce that

$$
t_{\mu \nu \rho \alpha}^{0}=0
$$

and

$$
t_{\mu \nu \rho \alpha \beta}^{1}=-t_{\mu \beta \rho \alpha \nu}^{1} .
$$

We may therefore write the amplitude as

$$
\mathcal{M}=\varepsilon_{g_{\mathrm{KK}}}^{\rho *}(r)\left(\varepsilon^{\nu}(q) q^{\beta}-\varepsilon^{\beta}(q) q^{\nu}\right)\left(\varepsilon^{\mu}(p) p^{\alpha}-\varepsilon^{\alpha}(p) p^{\mu}\right) t_{\mu \nu \rho \alpha \beta}^{1}(p, q),
$$

where $t_{\mu \nu \rho \alpha \beta}^{1}$ has the following properties:

- Does not contain $q_{\beta}, q_{\nu}$;

- Does not contain $p_{\alpha}, p_{\mu}$;

- Antisymmetric under $\alpha \leftrightarrow \mu$;

- Antisymmetric under $\beta \leftrightarrow \nu$; 
- We may exchange $p_{\rho} \leftrightarrow-q_{\rho}$ in any term.

There are 27 different combinations of $p, q$, the metric tensor $\eta$, and the Levi-Civita tensor $\epsilon$ that have these properties. The combinations are listed in appendix A. Having constructed such terms, we can consider the contribution to $F_{\mu \nu \rho}$ that each will provide. We note that, by construction, contraction according to equation (2.10) is equivalent to contraction of the contribution to $t_{\mu \nu \rho \alpha \beta}^{1}$ with $\varepsilon_{g_{\mathrm{KK}}}^{\rho}(r) \varepsilon^{\nu}(q) q^{\beta} \varepsilon^{\mu}(p) p^{\alpha}$, and we may therefore contract each contribution according to the equation

$$
F_{\mu \nu \rho}=p^{\alpha} q^{\beta} t_{\mu \nu \rho \alpha \beta}^{1} .
$$

Such a contraction yields four different forms that may contribute to $F_{\mu \nu \rho}$, namely

$$
\begin{gathered}
\left(\eta_{\mu \nu} p \cdot q-q_{\mu} p_{\nu}\right) p_{\rho}, \\
\epsilon_{\mu \nu \gamma \delta} p^{\gamma} q^{\delta} p_{\rho}, \\
\epsilon_{\mu \nu \rho \gamma} p^{\gamma} p \cdot q-\epsilon_{\mu \rho \gamma \delta} p^{\gamma} q^{\delta} p_{\nu}, \\
\epsilon_{\mu \nu \rho \gamma} q^{\gamma} p \cdot q-\epsilon_{\nu \rho \gamma \delta} p^{\gamma} q^{\delta} q_{\mu} .
\end{gathered}
$$

We may therefore write

$$
\begin{aligned}
& F_{\mu \nu \rho}=A\left(\eta_{\mu \nu} p \cdot q-q_{\mu} p_{\nu}\right) p_{\rho}+B \epsilon_{\mu \nu \gamma \delta} p^{\gamma} q^{\delta} p_{\rho}+ \\
& \quad+C\left(\epsilon_{\mu \nu \rho \gamma} p^{\gamma} p \cdot q-\epsilon_{\mu \rho \gamma \delta} p^{\gamma} q^{\delta} p_{\nu}\right)+D\left(\epsilon_{\mu \nu \rho \gamma} q^{\gamma} p \cdot q-\epsilon_{\nu \rho \gamma \delta} p^{\gamma} q^{\delta} q_{\mu}\right),
\end{aligned}
$$

where $A, B, C$ and $D$ are constants. The problem of calculating the amplitude reduces to the problem of calculating $A, B, C$ and $D$, and where two terms have the same coefficient, it suffices to evaluate the coefficient for one of them. This last observation will prove important later for simplifying the calculation.

\section{Feynman rules applicable to the calculation}

Many of the vertices and propagators applicable to the calculation are ones that appear in the Standard Model; however, it is necessary to add to the Feynman rules for those vertices and propagators rules for the KK gluon propagator and for its interactions.

Following references $[11,28]$, we recall that the KK gluon arises from a mode expansion of the components in the usual four dimensions of the five-dimensional gluon field into fields depending upon the standard four-dimensional coordinates $\left(A_{\mu}^{(n)}\left(x^{\mu}\right)\right)$ and fields depending upon the extra-dimensional coordinate $\left(\chi_{n}(\phi)\right)$. That is (following the conventions of reference [28]),

$$
A_{\mu}\left(x^{\mu}, \phi\right)=\sum_{n=0}^{\infty} A_{\mu}^{(n)}\left(x^{\mu}\right) \frac{\chi_{n}(\phi)}{\sqrt{R_{c}}},
$$

where $\phi=x^{4} / R_{c}$. The particle to which we refer as the "KK gluon" is the first excited mode $A_{\mu}^{(1)}$ - there are further excited modes, which we neglect as being suppressed by their higher masses. The mode decomposition leaves open in principle the possibility of a scalar 
gluon $A_{4}$, corresponding to the extra-dimensional component of the five-dimensional gluon. We shall eventually be constrained in our choice of gauge for $A_{4}$ by the requirement that the four-dimensional effective theory be anomaly-free ${ }^{1}$. However, we may begin by making the gauge choice $A_{4}=0$ for calculational convenience. Whilst the subsequent change of gauge affects some Feynman rules already used, the effects are loop-suppressed, so that their consideration is only necessary at two-loop level, and may be neglected at the one-loop level. Oscillations between KK modes are prevented by the orthonormality condition

$$
\int_{-\pi}^{\pi} d \phi \chi_{m} \chi_{n}=\delta^{m n}
$$

It is, however, notable that since there need be no momentum conservation in the extra dimension (there is not translational invariance), there is no a priori reason why there should not be interaction vertices between the Standard Model gluon and the KK gluon.

We may obtain the couplings at the interaction vertices (and, in particular, determine whether the couplings are non-zero) by integrating out the extra-dimensional wavefunctions $\chi_{n}$ that appear in the interaction terms. Reference [28] derives values for the wavefunctions of

$$
\begin{aligned}
& \chi_{0}=\frac{1}{\sqrt{2 \pi}}, \\
& \chi_{1}=\frac{e^{k R_{c}|\phi|}}{N_{1}}\left[J_{1}\left(\frac{M_{\mathrm{KK}}}{k} e^{k R_{c}|\phi|}\right)+\alpha_{1} Y_{1}\left(\frac{M_{\mathrm{KK}}}{k} e^{k R_{c}|\phi|}\right)\right],
\end{aligned}
$$

where $N_{1}$ is a normalisation constant, $\alpha_{1}$ is a constant and $J_{1}$ and $Y_{1}$ are Bessel functions of order 1. (The derivation is for an Abelian theory but holds in the non-Abelian case.) The couplings are determined by substituting equation (3.1) into the interacting part of the action (viz. $\left.-(1 / 4) F_{\mu \nu}\left(x^{\mu}, \phi\right) F^{\mu \nu}\left(x^{\mu}, \phi\right)\right)$ and integrating out the fifth dimensional component of the action $x_{4}=R_{c} \phi$.

For the Standard Model three-point coupling, this procedure gives us

$$
g=g_{5} \int_{-\pi}^{\pi} d \phi \frac{\chi_{0}^{3}}{\sqrt{R_{c}}}=\frac{g_{5}}{\sqrt{2 \pi R_{c}}}
$$

which we use to determine the relationship between the five-dimensional coupling $g_{5}$ and the Standard Model coupling $g$.

For the $g g g_{\mathrm{KK}}$ coupling, we note that all relevant terms in the action will yield the integral

$$
3 g_{5} \int_{-\pi}^{\pi} d \phi \frac{\chi_{0}^{2} \chi_{1}}{\sqrt{R_{c}}}=\frac{3 g_{5}}{\sqrt{2 \pi}} \int_{-\pi}^{\pi} d \phi \frac{\chi_{0} \chi_{1}}{\sqrt{R_{c}}}=0,
$$

where we have used equations (3.3) and (3.2) respectively for the two equalities. So, as already known, there is no $g g g_{\mathrm{KK}}$ vertex. By a similar argument, there is no $g g g g_{\mathrm{KK}}$ vertex.

\footnotetext{
${ }^{1}$ The five-dimensional theory is gauge-variant and consequently UV-divergent [29], but we can impose upon the four-dimensional theory a condition of gauge-invariance (or equivalently freedom from gauge anomalies) by our choice of five-dimensional gauge.
} 
For the $g g_{\mathrm{KK}} g_{\mathrm{KK}}$ coupling, the relevant terms in the action will yield the integral

$$
3 g_{5} \int_{-\pi}^{\pi} d \phi \frac{\chi_{0} \chi_{1}^{2}}{\sqrt{R_{c}}}=\frac{3 g_{5}}{\sqrt{2 \pi}} \int_{-\pi}^{\pi} d \phi \frac{\chi_{1}^{2}}{\sqrt{R_{c}}}=\frac{3 g_{5}}{\sqrt{2 \pi R_{c}}}=3 g
$$

where we have used equations (3.3), (3.2) and (3.5) respectively in the equalities. Noting that the symmetry factor for the $g g_{\mathrm{KK}} g_{\mathrm{KK}}$ vertex is 2 ! rather than the 3 ! of the $g g g$ vertex, we see that the two vertices have the same coupling.

The integral is very similar for the $g g g_{\mathrm{KK}} g_{\mathrm{KK}}$ coupling: we obtain

$$
3 !\left(g_{5}\right)^{2} \int_{-\pi}^{\pi} d \phi \frac{\chi_{0}^{2} \chi_{1}^{2}}{R_{c}}=\frac{3 !\left(g_{5}\right)^{2}}{2 \pi} \int_{-\pi}^{\pi} d \phi \frac{\chi_{1}^{2}}{R_{c}}=\frac{3 !\left(g_{5}\right)^{2}}{2 \pi R_{c}}=3 ! g^{2}
$$

again using equations (3.3), (3.2) and (3.5) respectively in the equalities. Noting that the symmetry factor for the $g g g_{\mathrm{KK}} g_{\mathrm{KK}}$ vertex is $2 ! \cdot 2$ ! rather than the 4 ! of the $g g g g$ vertex, we see that the two vertices have the same coupling.

The remaining gluon interaction vertices that we shall need are the $g_{\mathrm{KK}} g_{\mathrm{KK}} g_{\mathrm{KK}}$ and $g g_{\mathrm{KK}} g_{\mathrm{KK}} g_{\mathrm{KK}}$ vertices (the four-point $g_{\mathrm{KK}} g_{\mathrm{KK}} g_{\mathrm{KK}} g_{\mathrm{KK}}$ vertex is not required). To obtain these two vertices requires integrating $\chi_{1}^{3}$, which, given (3.4), is non-trivial. The approximations given in reference [28] are sufficient to allow a numerical integration [30,31], which shows the couplings to be non-zero and of the same orders of magnitude as their Standard Model counterparts (with $k R_{c}=11$, there is an enhancement of approximately 2.5 times). Since again the symmetry factors and Lagrangian multiplicities balance between the vertices and their Standard Model counterparts, we may write the couplings as $g^{(111)}$ for the $g_{\mathrm{KK}} g_{\mathrm{KK}} g_{\mathrm{KK}}$ coupling, and $g g^{(111)}$ for the $g g_{\mathrm{KK}} g_{\mathrm{KK}} g_{\mathrm{KK}}$ coupling, where $g^{(111)}$ depends upon the geometry of the extra dimension, but is approximately $2.5 \mathrm{~g}$ when $k R_{c}=11$.

The KK gluon propagator has the same structure as the Standard Model gluon propagator, but with a mass term. (We choose the Feynman gauge throughout.)

A one-loop calculation in Feynman gauge in principle can contain ghosts in diagrams. In order to provide the appropriate cancellations the ghosts must have a mode expansion in which the extra-dimensional component of the expansion is equal to that of the gluon component, i.e. $\chi_{n}$. This means that the values of the couplings of the ghost modes match the values of the couplings of the corresponding gluon modes. The propagator for the KK ghost has the same structure as the Standard Model ghost propagator, but with a mass term.

Finally, we shall need to consider the couplings between quarks and the KK gluon. These couplings vary with the bulk profiles of the quarks and with their handedness; we write the magnitude of the coupling as $g^{(1 q)}$ in each case and include a chiral projector in the Feynman rule.

The Feynman rules are summarized in figure 1. $f^{a b c}$ are the $\mathrm{SU}(3)$ anti-symmetric structure constants where $a, b, c, \ldots$ are adjoint $\mathrm{SU}(3)$ indices. $t^{a}$ are $\mathrm{SU}(3)$ generators and $A, B, C$ denote fundamental $\mathrm{SU}(3)$ indices. 
A,

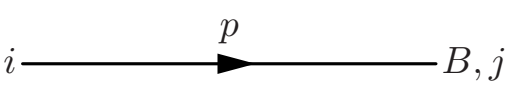

$\frac{i \delta^{A B}\left(\not p-m_{q}\right)_{j i}}{p^{2}-m_{q}^{2}}$

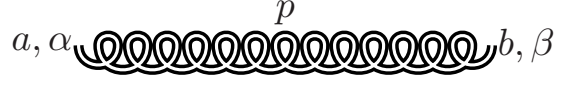

$$
\frac{-i \delta^{a b} \eta^{\alpha \beta}}{p^{2}-M_{\mathrm{KK}}^{2}}
$$

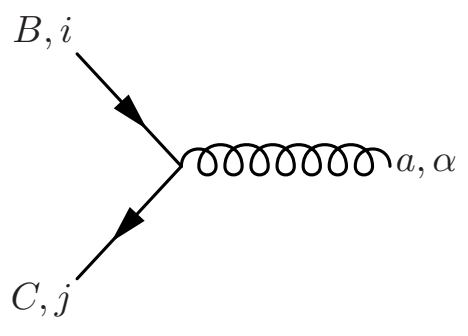

$$
-i g\left(t^{a}\right)_{\mathrm{CB}}\left(\gamma^{\alpha}\right)_{j i}
$$

$b, \beta$

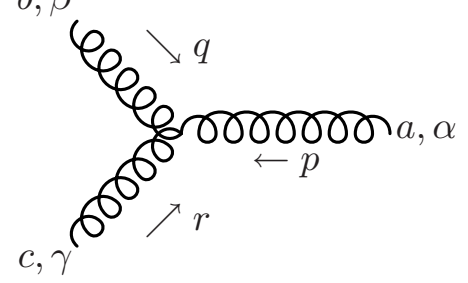

${ }_{a, \alpha}$ ebelebeleee ${ }^{b, \beta}$

$\frac{-i \delta^{a b} \eta^{\alpha \beta}}{p^{2}}$

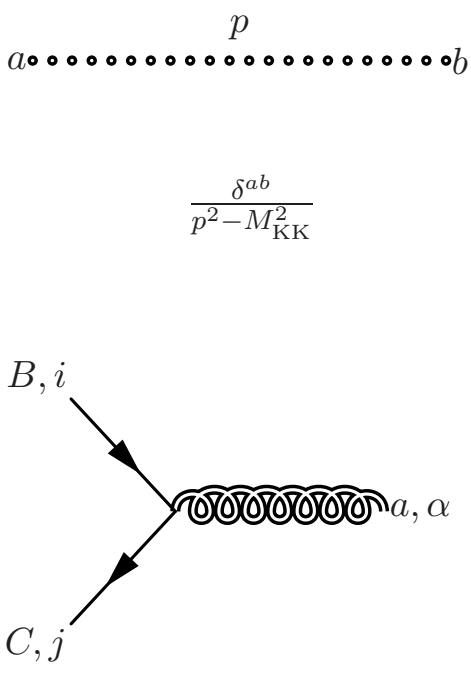

$-i g^{(1 q)}\left(t^{a}\right)_{\mathrm{CB}}\left(\gamma^{\alpha}\right)_{j k}\left(\left(1 \pm \gamma^{5}\right) / 2\right)_{k i}$

( \pm in chiral projector according to handedness of quark)

$$
\begin{aligned}
& b, \beta \text { (6) } q, \alpha \\
& c, \gamma=0 \\
& -g f^{a b c}\left[(p-q)^{\gamma} \eta^{\alpha \beta}+\right. \\
& +(q-r)^{\alpha} \eta^{\beta \gamma}+ \\
& \left.+(r-p)^{\beta} \eta^{\gamma \alpha}\right]
\end{aligned}
$$



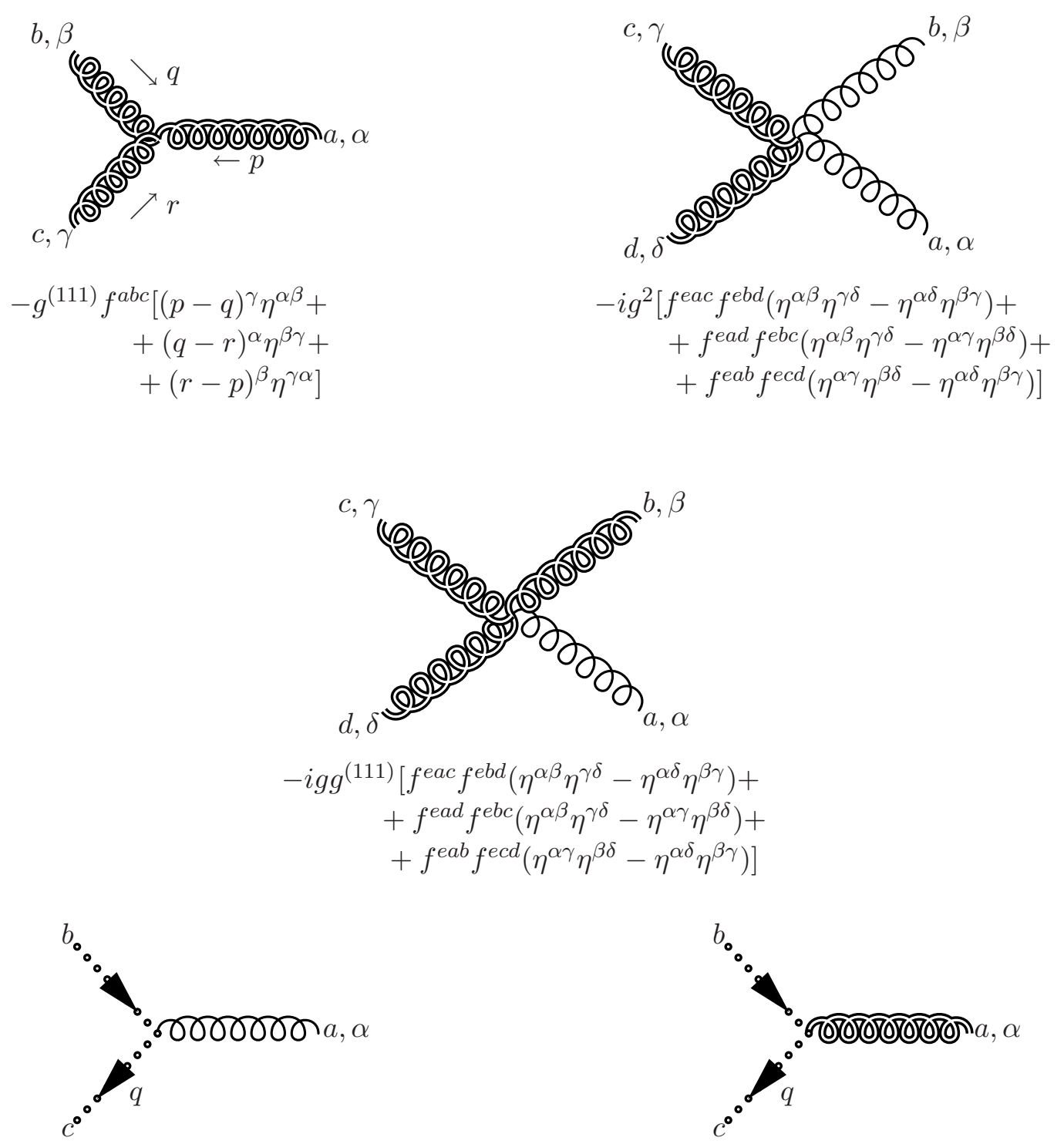

$$
g f^{a b c} q^{\alpha} \quad g^{(111)} f^{a b c} q^{\alpha}
$$

Figure 1. Feynman rules required to evaluate the diagrams relevant for the $g g \rightarrow g_{\mathrm{KK}}$ process. The KK gluon is denoted by a double gluon line, and the KK ghost is denoted by lines of circles.

\section{Diagrams}

\subsection{Quark loop diagrams}

Figure 2 contains diagrams for the process that have a quark in the loop. We may write the contributions to the amplitude from the individual diagrams as

$$
\begin{aligned}
F_{\mu \nu \rho}^{(q: a)}=- & \frac{1}{2} i g^{2} g^{(1 q)} f^{a d b} \delta^{d e} \operatorname{Tr}\left(t^{c} t^{e}\right)\left[\eta_{\mu \alpha}(p+r)_{\nu}+\eta_{\alpha \nu}(-r-q)_{\mu}+\eta_{\mu \nu}(q-p)_{\alpha}\right] \times \\
& \times \eta^{\alpha \beta} \frac{1}{r^{2}} \int \frac{d^{4} l}{(2 \pi)^{4}} \frac{\operatorname{Tr}\left[\gamma_{\beta}\left(l-m_{q}\right) \gamma_{\rho}\left(1 \pm \gamma^{5}\right)\left(l+\not-m_{q}\right)\right]}{\left[l^{2}-m_{q}^{2}\right]\left[(l+r)^{2}-m_{q}^{2}\right]},
\end{aligned}
$$




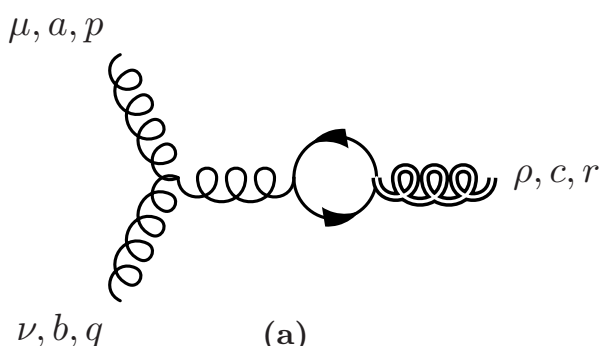

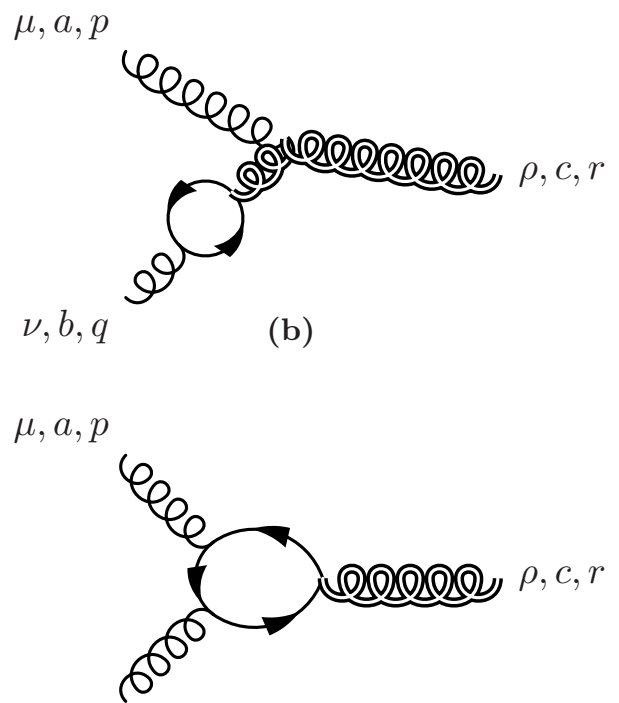

$\nu, b, q$

(d) $\mu, a, p$

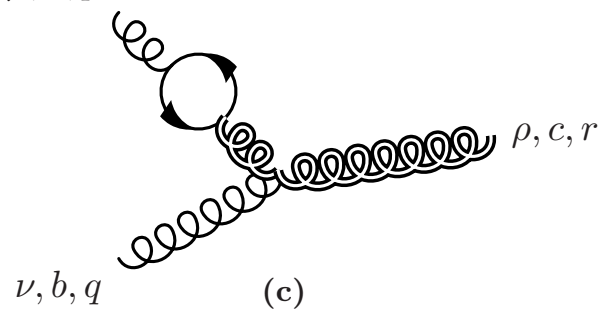

$\mu, a, p$

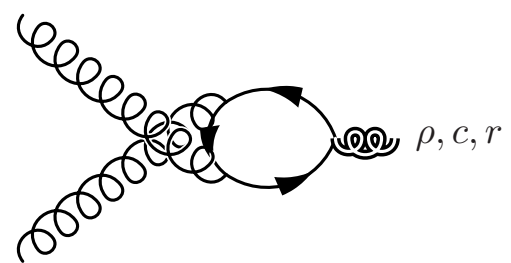

(e)

Figure 2. Feynman diagrams for the process that involve a quark in the loop.

$$
\begin{aligned}
F_{\mu \nu \rho}^{(q: b)}=- & \frac{1}{2} i g^{2} g^{(1 q)} \operatorname{Tr}\left(t^{b} t^{d}\right) \delta^{d e} f^{a e c} \frac{\eta^{\alpha \beta}}{q^{2}-M_{\mathrm{KK}}^{2}}\left[\eta_{\mu \beta}(p-q)_{\rho}+\eta_{\beta \rho}(q+r)_{\mu}+\eta_{\rho \mu}(-r-p)_{\beta}\right] \times \\
& \times \int \frac{d^{4} l}{(2 \pi)^{4}} \frac{\operatorname{Tr}\left[\gamma_{\nu}\left(l-m_{q}\right) \gamma_{\alpha}\left(1 \pm \gamma^{5}\right)\left(\not l+\not l-m_{q}\right)\right]}{\left[l^{2}-m_{q}^{2}\right]\left[(l+q)^{2}-m_{q}^{2}\right]}, \\
F_{\mu \nu \rho}^{(q: c)}=- & \frac{1}{2} i g^{2} g^{(1 q)} \operatorname{Tr}\left(t^{a} t^{d}\right) \delta^{d e} f^{b e c} \frac{\eta^{\alpha \beta}}{p^{2}-M_{\mathrm{KK}}^{2}}\left[\eta_{\nu \beta}(q-p)_{\rho}+\eta_{\beta \rho}(p+r)_{\nu}+\eta_{\rho \nu}(-r-q)_{\beta}\right] \times \\
& \times \int \frac{d^{4} l}{(2 \pi)^{4}} \frac{\operatorname{Tr}\left[\gamma_{\mu}\left(\not l-m_{q}\right) \gamma_{\alpha}\left(1 \pm \gamma^{5}\right)\left(l+\not p-m_{q}\right)\right]}{\left[l^{2}-m_{q}^{2}\right]\left[(l+p)^{2}-m_{q}^{2}\right]} \\
F_{\mu \nu \rho}^{(q: d)=-} & \frac{1}{2} g^{2} g^{(1 q)} \operatorname{Tr}\left(t^{a} t^{c} t^{b}\right) \times \\
& \times \int \frac{d^{4} l}{(2 \pi)^{4}} \frac{\operatorname{Tr}\left[\gamma_{\mu}\left(\not l-\not p-m_{q}\right) \gamma_{\rho}\left(1 \pm \gamma^{5}\right)\left(\not l+\not l-m_{q}\right) \gamma_{\nu}\left(l-m_{q}\right)\right]}{\left[(l-p)^{2}-m_{q}^{2}\right]\left[(l+q)^{2}-m_{q}^{2}\right]\left[l^{2}-m_{q}^{2}\right]} \\
F_{\mu \nu \rho}^{(q: e)=-} & \frac{1}{2} g^{2} g^{(1 q)} \operatorname{Tr}\left(t^{b} t^{c} t^{a}\right) \times \\
& \times \int \frac{d^{4} l}{(2 \pi)^{4}} \frac{\operatorname{Tr}\left[\gamma_{\nu}\left(l-\not l-m_{q}\right) \gamma_{\rho}\left(1 \pm \gamma^{5}\right)\left(l+\not p-m_{q}\right) \gamma_{\mu}\left(l-m_{q}\right)\right]}{\left[(l-q)^{2}-m_{q}^{2}\right]\left[(l+p)^{2}-m_{q}^{2}\right]\left[l^{2}-m_{q}^{2}\right]}
\end{aligned}
$$


denoting the contribution from quark loop diagram $a$ by $F_{\mu \nu \rho}^{(q: a)}$, etc. These contributions are applicable to KK quarks as well, with appropriate modification of couplings.

\subsection{Kaluza-Klein gluon (and ghost) loop diagrams}

There are no diagrams with a gluon in the loop, since there is no vertex containing gluons and a single KK gluon. However, there are diagrams with KK gluons (and KK ghosts) in the loop. These diagrams are shown in figures 3 and 4 .

We may write the contributions to the amplitude from the KK gluon diagrams as

$$
\begin{aligned}
& F_{\mu \nu \rho}^{(g: a)}=-i g^{2} g^{(111)} f^{a b d} f^{e f i} f^{c h g} \delta^{d e} \delta^{f g} \delta^{h i}\left[(p-q)_{\alpha} \eta_{\mu \nu}+(q+r)_{\mu} \eta_{\nu \alpha}+(-r-p)_{\nu} \eta_{\mu \alpha}\right] \times \\
& \times \frac{1}{r^{2}} \eta^{\alpha \beta} \eta^{\gamma \delta} \eta^{\epsilon \zeta} \int \frac{d^{4} l}{(2 \pi)^{4}}\left\{\frac{1}{\left[(l+r)^{2}-M_{\mathrm{KK}}^{2}\right]\left[l^{2}-M_{\mathrm{KK}}^{2}\right]} \times\right. \\
& \times\left[(2 r+l)_{\zeta} \eta_{\beta \gamma}+(-2 l-r)_{\beta} \eta_{\gamma \zeta}+(l-r)_{\gamma} \eta_{\beta \zeta}\right] \times \\
& \left.\times\left[(-r+l)_{\delta} \eta_{\epsilon \rho}+(-2 l-r)_{\rho} \eta_{\epsilon \delta}+(l+2 r)_{\epsilon} \eta_{\rho \delta}\right]\right\}, \\
& F_{\mu \nu \rho}^{(g: b)}=-i g^{2} g^{(111)} f^{c a d} f^{e f i} f^{b h g} \delta^{d e} \delta^{f g} \delta^{h i}\left[(-r-p)_{\alpha} \eta_{\rho \mu}+(p-q)_{\rho} \eta_{\mu \alpha}+(q+r)_{\mu} \eta_{\rho \alpha}\right] \times \\
& \times \frac{1}{q^{2}-M_{\mathrm{KK}}^{2}} \eta^{\alpha \beta} \eta^{\gamma \delta} \eta^{\epsilon \zeta} \int \frac{d^{4} l}{(2 \pi)^{4}}\left\{\frac{1}{\left[(l-q)^{2}-M_{\mathrm{KK}}^{2}\right]\left[l^{2}-M_{\mathrm{KK}}^{2}\right]} \times\right. \\
& \times\left[(-2 q+l)_{\zeta} \eta_{\beta \gamma}+(q-2 l)_{\beta} \eta_{\gamma \zeta}+(l+q)_{\gamma} \eta_{\beta \zeta}\right] \times \\
& \left.\times\left[(q+l)_{\delta} \eta_{\epsilon \nu}+(q-2 l)_{\nu} \eta_{\epsilon \delta}+(l-2 q)_{\epsilon} \eta_{\nu \delta}\right]\right\}
\end{aligned}
$$

$F_{\mu \nu \rho}^{(g: c)}=-i g^{2} g^{(111)} f^{c b d} f^{e f i} f^{a h g} \delta^{d e} \delta^{f g} \delta^{h i}\left[(-r-q)_{\alpha} \eta_{\rho \nu}+(q-p)_{\rho} \eta_{\nu \alpha}+(p+r)_{\nu} \eta_{\rho \alpha}\right] \times$ $\times \frac{1}{p^{2}-M_{\mathrm{KK}}^{2}} \eta^{\alpha \beta} \eta^{\gamma \delta} \eta^{\epsilon \zeta} \int \frac{d^{4} l}{(2 \pi)^{4}}\left\{\frac{1}{\left[(l-p)^{2}-M_{\mathrm{KK}}^{2}\right]\left[l^{2}-M_{\mathrm{KK}}^{2}\right]} \times\right.$$$
\times\left[(-2 p+l)_{\zeta} \eta_{\beta \gamma}+(p-2 l)_{\beta} \eta_{\gamma \zeta}+(l+p)_{\gamma} \eta_{\beta \zeta}\right] \times
$$$$
\left.\times\left[(p+l)_{\delta} \eta_{\epsilon \mu}+(p-2 l)_{\mu} \eta_{\epsilon \delta}+(l-2 p)_{\epsilon} \eta_{\mu \delta}\right]\right\}
$$

$$
\begin{aligned}
F_{\mu \nu \rho}^{(g: d)}= & -i g^{2} g^{(111)} \frac{1}{r^{2}} \eta^{\alpha \beta} \eta^{\gamma \delta} f^{a b d}\left[(p-q)_{\alpha} \eta_{\mu \nu}+(q+r)_{\mu} \eta_{\nu \alpha}+(-r-p)_{\nu} \eta_{\mu \alpha}\right] \delta^{d e} \delta^{f g} \times \\
\times & {\left[f^{x e g} f^{x f c}\left(\eta_{\beta \gamma} \eta_{\delta \rho}-\eta_{\beta \rho} \eta_{\gamma \delta}\right)+f^{x e c} f^{x f g}\left(\eta_{\beta \gamma} \eta_{\delta \rho}-\eta_{\beta \delta} \eta_{\gamma \rho}\right)+\right.} \\
& \left.\quad+f^{x e f} f^{x g c}\left(\eta_{\beta \delta} \eta_{\gamma \rho}-\eta_{\beta \rho} \eta_{\gamma \delta}\right)\right] \int \frac{d^{4} l}{(2 \pi)^{4}} \frac{1}{l^{2}-M_{\mathrm{KK}}^{2}}
\end{aligned}
$$

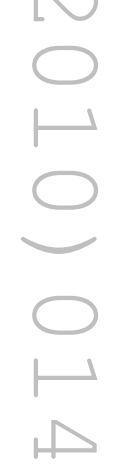




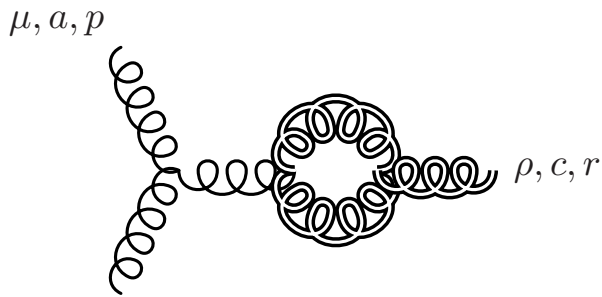

(a)

$\mu, a, p$

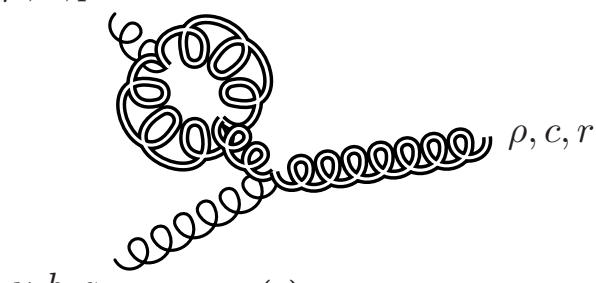

(c)

$\mu, a, p$

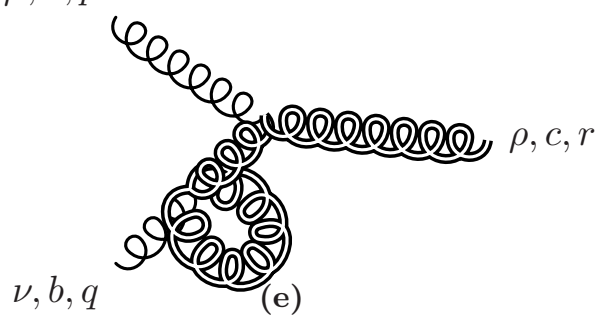

$\mu, a, p$

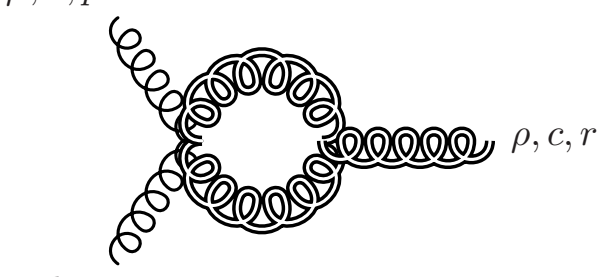

$\nu, b, q$

(g)

$\mu, a, p$

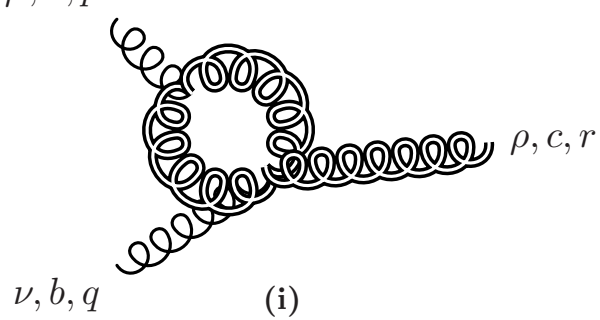

$\mu, a, p$

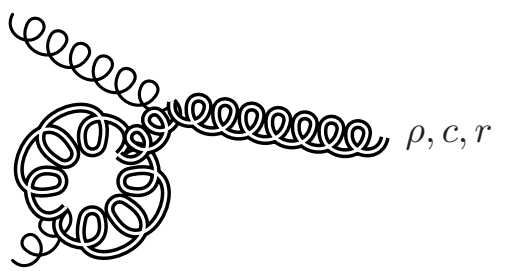

(b)

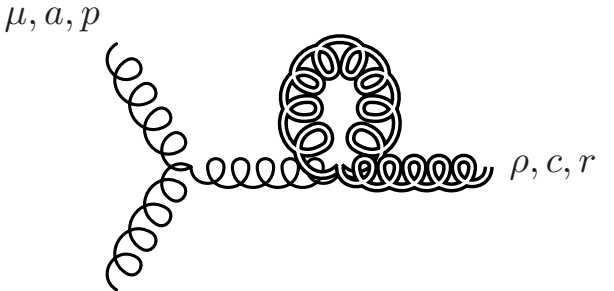

$\nu, b, q \quad$ (d)
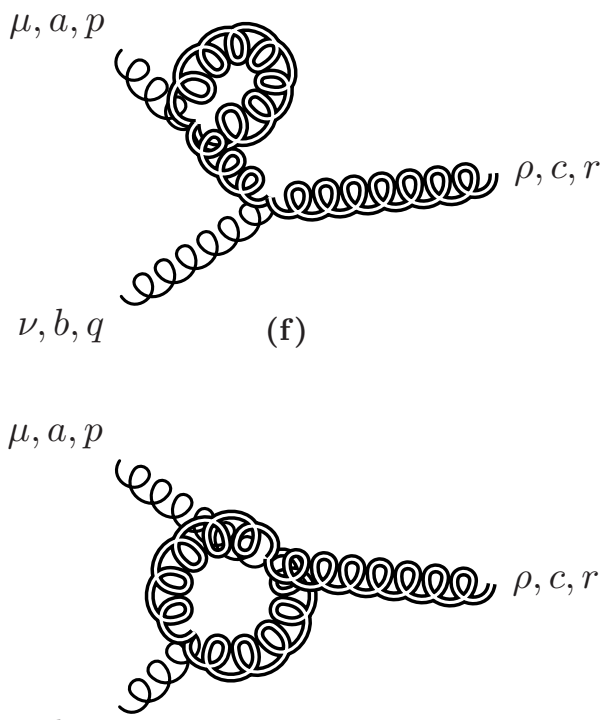

$\nu, b, q \quad$ (h)

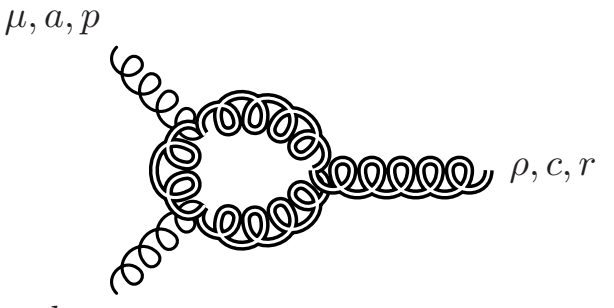

$\nu, b, q$

(j)

Figure 3. Feynman diagrams for the process that involve a Kaluza-Klein gluon in the loop. 


$$
\begin{aligned}
& F_{\mu \nu \rho}^{(g: e)}=-i g^{2} g^{(111)} \frac{1}{q^{2}-M_{\mathrm{KK}}^{2}} \eta^{\alpha \beta} \eta^{\gamma \delta} f^{c a d}\left[(-r-p)_{\alpha} \eta_{\rho \mu}+(p-q)_{\rho} \eta_{\mu \alpha}+(q+r)_{\mu} \eta_{\rho \alpha}\right] \times \\
& \times \delta^{d e} \delta^{f g}\left[f^{x e g} f^{x f b}\left(\eta_{\beta \gamma} \eta_{\delta \nu}-\eta_{\beta \nu} \eta_{\gamma \delta}\right)+f^{x e b} f^{x f g}\left(\eta_{\beta \gamma} \eta_{\delta \nu}-\eta_{\beta \delta} \eta_{\gamma \nu}\right)+\right. \\
& \left.+f^{x e f} f^{x g b}\left(\eta_{\beta \delta} \eta_{\gamma \nu}-\eta_{\beta \nu} \eta_{\gamma \delta}\right)\right] \int \frac{d^{4} l}{(2 \pi)^{4}} \frac{1}{l^{2}-M_{\mathrm{KK}}^{2}}, \\
& F_{\mu \nu \rho}^{(g: f)}=-i g^{2} g^{(111)} \frac{1}{p^{2}-M_{\mathrm{KK}}^{2}} \eta^{\alpha \beta} \eta^{\gamma \delta} f^{c b d}\left[(-r-q)_{\alpha} \eta_{\rho \nu}+(q-p)_{\rho} \eta_{\nu \alpha}+(p+r)_{\nu} \eta_{\rho \alpha}\right] \times \\
& \times \delta^{d e} \delta^{f g}\left[f^{x e g} f^{x f a}\left(\eta_{\beta \gamma} \eta_{\delta \mu}-\eta_{\beta \mu} \eta_{\gamma \delta}\right)+f^{x e a} f^{x f g}\left(\eta_{\beta \gamma} \eta_{\delta \mu}-\eta_{\beta \delta} \eta_{\gamma \mu}\right)+\right. \\
& \left.+f^{x e f} f^{x g a}\left(\eta_{\beta \delta} \eta_{\gamma \mu}-\eta_{\beta \mu} \eta_{\gamma \delta}\right)\right] \int \frac{d^{4} l}{(2 \pi)^{4}} \frac{1}{l^{2}-M_{\mathrm{KK}}^{2}}, \\
& F_{\mu \nu \rho}^{(g: g)}=-i g^{2} g^{(111)} f^{c f e} \delta^{d e} \delta^{f g} \eta^{\alpha \beta} \eta^{\gamma \delta} \times \\
& \times\left[f ^ { x a d } f ^ { x b g } \left(\eta_{\mu \nu} \eta_{\alpha \delta}-\eta_{\mu \delta} \eta_{\nu \alpha}+f^{x a g} f^{x b d}\left(\eta_{\mu \nu} \eta_{\alpha \delta}-\eta_{\mu \alpha} \eta_{\nu \delta}\right)+\right.\right. \\
& \left.f^{x a b} f^{x d g}\left(\eta_{\mu \alpha} \eta_{\nu \delta}-\eta_{\mu \delta} \eta_{\nu \alpha}\right)\right] \times \\
& \times \int \frac{d^{4} l}{(2 \pi)^{4}} \frac{\left[(l-r)_{\beta} \eta_{\rho \gamma}+(-2 l-r)_{\rho} \eta_{\gamma \beta}+(l+2 r)_{\gamma} \eta_{\beta \rho}\right]}{\left[(l+r)^{2}-M_{\mathrm{KK}}^{2}\right]\left[l^{2}-M_{\mathrm{KK}}^{2}\right]}, \\
& F_{\mu \nu \rho}^{(g: h)}=-i g^{2} g^{(111)} f^{b f e} \delta^{d e} \delta^{f g} \eta^{\alpha \beta} \eta^{\gamma \delta} \times \\
& \times\left[f ^ { x c d } f ^ { x a g } \left(\eta_{\rho \mu} \eta_{\alpha \delta}-\eta_{\rho \delta} \eta_{\mu \alpha}+f^{x c g} f^{x a d}\left(\eta_{\rho \mu} \eta_{\alpha \delta}-\eta_{\rho \alpha} \eta_{\mu \delta}\right)+\right.\right. \\
& \left.f^{x c b} f^{x a g}\left(\eta_{\rho \alpha} \eta_{\mu \delta}-\eta_{\rho \delta} \eta_{\mu \alpha}\right)\right] \times \\
& \times \int \frac{d^{4} l}{(2 \pi)^{4}} \frac{\left[(l+q)_{\beta} \eta_{\nu \gamma}+(q-2 l)_{\nu} \eta_{\gamma \beta}+(l-2 q)_{\gamma} \eta_{\beta \nu}\right]}{\left[(l-q)^{2}-M_{\mathrm{KK}}^{2}\right]\left[l^{2}-M_{\mathrm{KK}}^{2}\right]}, \\
& F_{\mu \nu \rho}^{(g: i)}=-i g^{2} g^{(111)} f^{a f e} \delta^{d e} \delta^{f g} \eta^{\alpha \beta} \eta^{\gamma \delta} \times \\
& \times\left[f ^ { x c d } f ^ { x b g } \left(\eta_{\rho \nu} \eta_{\alpha \delta}-\eta_{\rho \delta} \eta_{\nu \alpha}+f^{x c g} f^{x b d}\left(\eta_{\rho \nu} \eta_{\alpha \delta}-\eta_{\rho \alpha} \eta_{\nu \delta}\right)+\right.\right. \\
& \left.f^{x c b} f^{x d g}\left(\eta_{\rho \alpha} \eta_{\nu \delta}-\eta_{\rho \delta} \eta_{\nu \alpha}\right)\right] \times \\
& \times \int \frac{d^{4} l}{(2 \pi)^{4}} \frac{\left[(l+p)_{\beta} \eta_{\mu \gamma}+(p-2 l)_{\mu} \eta_{\gamma \beta}+(l-2 p)_{\gamma} \eta_{\beta \mu}\right]}{\left[(l-p)^{2}-M_{\mathrm{KK}}^{2}\right]\left[l^{2}-M_{\mathrm{KK}}^{2}\right]}, \\
& F_{\mu \nu \rho}^{(g: j)}=-i g^{2} g^{(111)} f^{a d i} f^{c f e} f^{b h g} \delta^{d e} \delta^{f g} \delta^{h i} \eta^{\alpha \beta} \eta^{\gamma \delta} \eta^{\epsilon \zeta} \times \\
& \times \int \frac{d^{4} l}{(2 \pi)^{4}} \frac{1}{\left[(l+p)^{2}-M_{\mathrm{KK}}^{2}\right]\left[(l-q)^{2}-M_{\mathrm{KK}}^{2}\right]\left[l^{2}-M_{\mathrm{KK}}^{2}\right]} \times \\
& \times\left[(2 p+l)_{\zeta} \eta_{\alpha \mu}+(-2 l-p)_{\mu} \eta_{\alpha \zeta}+(l-p)_{\alpha} \eta_{\mu \zeta}\right] \times \\
& \times\left[(-r+l-q)_{\beta} \eta_{\rho \gamma}+(q-2 l-p)_{\rho} \eta_{\beta \gamma}+(l+p+r)_{\gamma} \eta_{\rho \beta}\right] \times \\
& \times\left[(q+l)_{\delta} \eta_{\epsilon \nu}+(-2 l+q)_{\nu} \eta_{\epsilon \delta}+(l-2 q)_{\epsilon} \eta_{\nu \delta}\right]
\end{aligned}
$$

denoting the contribution from KK gluon loop diagram $a$ by $F_{\mu \nu \rho}^{(g: a)}$, etc. We may write the contributions to the amplitude from the KK ghost diagrams as 


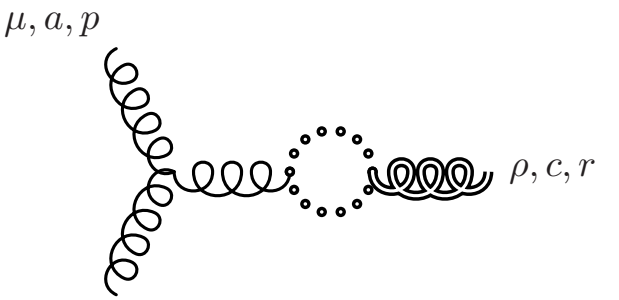

$\nu, b, q$

(a)

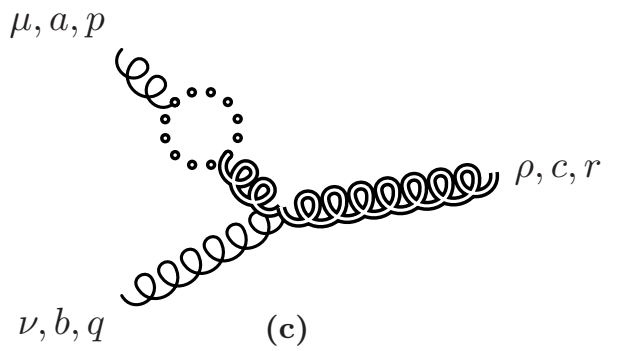

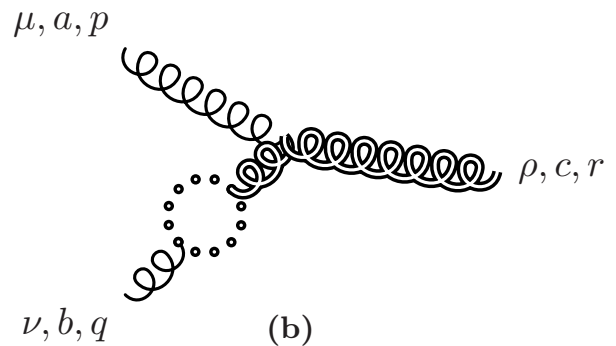

$\mu, a, p$

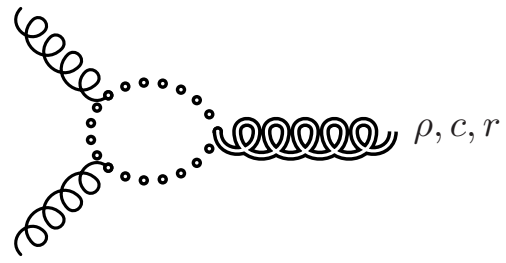

(d)

Figure 4. Feynman diagrams for the process that involve a Kaluza-Klein ghost in the loop.

$$
\begin{aligned}
F_{\mu \nu \rho}^{(\mathrm{gh}: a)}= & i g^{2} g^{(111)} f^{a d b} f^{c g h} f^{e i f} \delta^{d e} \delta^{f g} \delta^{h i}\left[(p+r)_{\nu} \eta_{\mu \alpha}+(-r-q)_{\mu} \eta_{\nu \alpha}+(q-p)_{\alpha} \eta_{\mu \nu}\right] \times \\
& \times \frac{1}{r^{2}} \eta^{\alpha \beta} \int \frac{d^{4} l}{(2 \pi)^{4}} \frac{(l+r)_{\rho} l_{\beta}}{\left[(l+r)^{2}-M_{\mathrm{KK}}^{2}\right]\left[l^{2}-M_{\mathrm{KK}}^{2}\right]}, \\
F_{\mu \nu \rho}^{(\text {gh: }: b)}= & i g^{2} g^{(111)} f^{a e c} f^{d i f} f^{b g h} \delta^{d e} \delta^{f g} \delta^{h i}\left[(p-q)_{\rho} \eta_{\beta \mu}+(q+r)_{\mu} \eta_{\beta \rho}+(-r-p)_{\beta} \eta_{\rho \mu}\right] \times \\
& \times \frac{1}{q^{2}-M_{\mathrm{KK}}^{2}} \eta^{\alpha \beta} \int \frac{d^{4} l}{(2 \pi)^{4}} \frac{l_{\alpha}(l+q)_{\nu}}{\left[l^{2}-M_{\mathrm{KK}}^{2}\right]\left[(l+q)^{2}-M_{\mathrm{KK}}^{2}\right]}, \\
F_{\mu \nu \rho}^{(\text {gh: } c)}= & i g^{2} g^{(111)} f^{b e c} f^{d i f} f^{a g h} \delta^{d e} \delta^{f g} \delta^{h i}\left[(q-p)_{\rho} \eta_{\beta \nu}+(p+r)_{\nu} \eta_{\beta \rho}+(-r-q)_{\beta} \eta_{\rho \nu}\right] \times \\
& \times \frac{1}{p^{2}-M_{\mathrm{KK}}^{2}} \eta^{\alpha \beta} \int \frac{d^{4} l}{(2 \pi)^{4}} \frac{l_{\alpha}(l+p)_{\nu}}{\left[l^{2}-M_{\mathrm{KK}}^{2}\right]\left[(l+p)^{2}-M_{\mathrm{KK}}^{2}\right]} \\
F_{\mu \nu \rho}^{(\text {gh: } d)}= & i g^{2} g^{(111)} f^{a i d} f^{c e f} f^{b g h} \delta^{d e} \delta^{f g} \delta^{h i} \times \\
& \times \int \frac{d^{4} l}{(2 \pi)^{4}} \frac{(l+p)_{\mu}(l-q)_{\rho} l_{\nu}}{\left[(l+p)^{2}-M_{\mathrm{KK}}^{2}\right]\left[(l-q)^{2}-M_{\mathrm{KK}}^{2}\right]\left[l^{2}-M_{\mathrm{KK}}^{2}\right]}
\end{aligned}
$$

denoting the contribution from KK ghost loop diagram $a$ by $F_{\mu \nu \rho}^{(\text {gh: } a)}$, etc.

\subsection{Counterterm diagrams}

If we are to use bare parameters in the expressions for the diagrams given so far, then diagrams containing counterterms will appear to balance the divergences from the previous diagrams. However, we shall see that it is possible to avoid considering such diagrams, and so we shall not detail them in full here. It suffices to observe that there are four counterterm diagrams (their appearance is that of the KK ghost loop diagrams in figure 4, with each KK ghost loop replaced by a counterterm), and that the Lorentz structure of the counterterms is derived from consideration of the underlying Lagrangian term. In 
particular, each term in the three-point counterterm vertex has one momentum factor carrying a Lorentz index (the other two being carried by a metric factor), and the two-point counterterm vertex has the sum of a term where the metric carries both external Lorentz indices and a term where there are two momentum terms each carrying an external Lorentz index. We note that this latter term always contains a momentum factor that contracts with an external polarisation vector to give zero. It is therefore the case that none of the counterterm diagrams contains a term where there is more than one momentum factor carrying an external Lorentz index. (There is also no term with a Levi-Civita tensor carrying an external Lorentz index.)

\section{Calculation of the amplitude}

\subsection{Simplification of the calculation}

Before proceeding to calculate the $g g \rightarrow g_{\mathrm{KK}}$ amplitude, we note that we can simplify our calculation significantly by using the general form derived in equation (2.16) to justify disregarding many diagrams.

Firstly, we note that the only diagrams capable of producing a Levi-Civita tensor are those containing a trace of a $\gamma^{5}$, i.e. the diagrams with quark loops. Of the diagrams with quark loops, we note that the loop integrals for diagrams (a), (b) and (c) only contain the loop momentum and one other momentum, and have as a maximum two factors of the momentum on the numerator (both of which contract with a trace of gamma matrices). This means that, even taking reparametrisation of the integrand into account, the only possible terms in the numerator contain either

- Two identical momenta contracted with a Levi-Civita tensor, which gives zero since the Levi-Civita tensor is antisymmetric, or

- One loop momentum and one other momentum contracted with a Levi-Civita tensor, which gives zero since such a term is odd in the loop momentum and the loop momentum integral is over all of space-time, or

- No loop momenta, but such a term does not yield a Levi-Civita tensor, since the trace involving a $\gamma^{5}$ term contains only two other gamma matrices, and this is zero.

So the only contributions to amplitude coefficients $B, C$ and $D$ come from $F_{\mu \nu \rho}^{(q: d)}$ and $F_{\mu \nu \rho}^{(q: e)}$.

Secondly, we note that in evaluating the contribution to amplitude coefficient $A$, we may sum the coefficients of either the term $\eta_{\mu \nu} p \cdot q p_{\rho}$ or the term $-q_{\mu} p_{\nu} p_{\rho}$. We choose the latter term.

We have already noted that no counterterm diagram contains more than one loop momentum carrying an external Lorentz index, so no counterterm diagram provides a contribution we need to evaluate.

In the quark loop sector, it initially appears that we can obtain a contribution we need to evaluate from each diagram, it being possible to obtain terms with three external momenta carrying external Lorentz indices in each case. However, we note that considering 
the loop momentum integral, in the case of diagram (a) such a term would have to contain a factor of $r_{\rho}$, which contracts with the external polarisation vector to give zero, and similarly such a term in diagram (b) would have to contain a factor of $q_{\nu}$ and such a term in diagram (c) would have to contain a factor of $p_{\mu}$, both of which contract with external polarisation vectors to give zero. So the only contributions to amplitude coefficient $A$ from the quark loop sector that we need to evaluate come from $F_{\mu \nu \rho}^{(q: d)}$ and $F_{\mu \nu \rho}^{(q: e)}$.

Similarly, whilst in the KK gluon loop sector, it initially appears that we can obtain a contribution we need to evaluate from diagrams (a), (b), (c) and (j), we note that the terms with three external momenta carrying external Lorentz indices in diagrams (a), (b) and (c) contain factors of $r_{\rho}, q_{\nu}$ and $p_{\mu}$ respectively, all of which contract with external polarisation vectors to give zero. So the only contribution to amplitude coefficient $A$ from the KK gluon loop sector that we need to evaluate comes from $F_{\mu \nu \rho}^{(g: j)}$.

The behaviour of the KK ghost loop sector is similar to that of the quark loop sector, and the only contribution to the amplitude coefficient $A$ from the KK ghost loop sector that we need to evaluate comes from $F_{\mu \nu \rho}^{(\text {gh: } d)}$.

We have therefore reduced the calculations required to derive the amplitude to those required to deduce the coefficients of single Levi-Civita tensors and of the term $-q_{\mu} p_{\nu} p_{\rho}$ in $F_{\mu \nu \rho}^{(q: d)}+F_{\mu \nu \rho}^{(q: e)}$, and of the term $-q_{\mu} p_{\nu} p_{\rho}$ in $F_{\mu \nu \rho}^{(g: j)}+F_{\mu \nu \rho}^{(\text {gh:d) }}$.

\subsection{Contribution from diagrams with quark loops}

We have established that we only need to consider diagrams (d) and (e) in the quark loop sector, and noting the similarities in their structure we begin by attempting to sum the diagrams without evaluating them.

Firstly, we note that we can apply recursively the identity

$$
t^{a} t^{b}=\frac{1}{6} \delta^{a b} I_{3}+\frac{1}{2}\left(i f^{a b c}+d^{a b c}\right) t^{c}
$$

along with the property that the $t^{a}$ are traceless, to deduce that

$$
\operatorname{Tr}\left(t^{a} t^{b} t^{c}\right)=\frac{1}{4}\left(i f^{a b c}+d^{a b c}\right) .
$$

Next, we note that we can apply the charge conjugation relations

$$
C^{-1} \gamma^{\mu} C=-\gamma^{\mu T}, \quad C^{-1} \gamma^{\mu} \gamma^{5} C=\left(\gamma^{\mu} \gamma^{5}\right)^{T},
$$

along with the cyclic property of the trace and the trace reversal property of the transpose, to obtain

$$
\begin{gathered}
\int \frac{d^{4} l}{(2 \pi)^{4}} \frac{\operatorname{Tr}\left[\gamma_{\nu}\left(\not l-\not l-m_{q}\right) \gamma_{\rho}\left(1 \pm \gamma^{5}\right)\left(\not l+\not p-m_{q}\right) \gamma_{\mu}\left(\not l-m_{q}\right)\right]}{\left[(l-q)^{2}-m_{q}^{2}\right]\left[(l+p)^{2}-m_{q}^{2}\right]\left[l^{2}-m_{q}^{2}\right]}= \\
=\int \frac{d^{4} l}{(2 \pi)^{4}} \frac{\operatorname{Tr}\left[\left(-\not l-m_{q}\right)\left(-\gamma_{\mu}\right)\left(-\not l-\not p-m_{q}\right) \gamma_{\rho}\left(-1 \pm \gamma^{5}\right)\left(-\not l+\not l-m_{q}\right)\left(-\gamma_{\nu}\right)\right]}{\left[(l-q)^{2}-m_{q}^{2}\right]\left[(l+p)^{2}-m_{q}^{2}\right]\left[l^{2}-m_{q}^{2}\right]}= \\
=\int \frac{d^{4} l}{(2 \pi)^{4}} \frac{\operatorname{Tr}\left[\left(\not l-m_{q}\right) \gamma_{\mu}\left(\not l-\not p-m_{q}\right) \gamma_{\rho}\left(1 \mp \gamma^{5}\right)\left(\not l+\not l-m_{q}\right) \gamma_{\nu}\right]}{\left[(l+q)^{2}-m_{q}^{2}\right]\left[(l-p)^{2}-m_{q}^{2}\right]\left[l^{2}-m_{q}^{2}\right]}
\end{gathered}
$$


where we have taken $l \rightarrow-l$ in the final line.

Substituting equations (5.2) and (5.4) into equations (4.4) and (4.5), we obtain

$$
\begin{aligned}
F_{\mu \nu \rho}^{(q: d)}+F_{\mu \nu \rho}^{(q: e)}= & -\frac{1}{4} g^{2} g^{(1 q)} i f^{a c b} \int \frac{d^{4} l}{(2 \pi)^{4}} \frac{\operatorname{Tr}\left[\gamma_{\mu}\left(l-\not p-m_{q}\right) \gamma_{\rho}\left(\not l+\not l-m_{q}\right) \gamma_{\nu}\left(l-m_{q}\right)\right]}{\left[(l-p)^{2}-m_{q}^{2}\right]\left[(l+q)^{2}-m_{q}^{2}\right]\left[l^{2}-m_{q}^{2}\right]} \mp \\
& \mp \frac{1}{4} g^{2} g^{(1 q)} d^{a c b} \int \frac{d^{4} l}{(2 \pi)^{4}} \frac{\operatorname{Tr}\left[\gamma_{\mu}\left(\not l-\not p-m_{q}\right) \gamma_{\rho} \gamma^{5}\left(\not l+\not l-m_{q}\right) \gamma_{\nu}\left(l-m_{q}\right)\right]}{\left[(l-p)^{2}-m_{q}^{2}\right]\left[(l+q)^{2}-m_{q}^{2}\right]\left[l^{2}-m_{q}^{2}\right]} .
\end{aligned}
$$

Applying a Feynman parametrisation and integral redefinition

$$
\begin{aligned}
\int \frac{d^{4} l}{(2 \pi)^{4}} \frac{f(l)}{\left[(l-p)^{2}-m_{q}^{2}\right]\left[(l+q)^{2}-m_{q}^{2}\right]\left[l^{2}-m_{q}^{2}\right]}= \\
=\int_{0}^{1} d x \int_{0}^{1-x} d y \int \frac{d^{4} l}{(2 \pi)^{4}} \frac{f(l+x p-y q)}{\left[l^{2}+2 x y p \cdot q-m_{q}^{2}\right]^{3}},
\end{aligned}
$$

where we have used equation $(1.5)\left(p^{2}=q^{2}=0\right)$ to simplify the final denominator, and keeping from the first integral of equation (5.5) only the terms parallel to $-q_{\mu} p_{\nu} p_{\rho}$ and from the second integral of the equation only the terms parallel to $\epsilon_{\mu \nu \gamma \delta} p^{\gamma} q^{\delta} p_{\rho}, \epsilon_{\mu \rho \gamma \delta} p^{\gamma} q^{\delta} p_{\nu}$, and $\epsilon_{\nu \rho \gamma \delta} p^{\gamma} q^{\delta} q_{\mu}$ (these are the only terms required to evaluate the coefficients $A, B, C$ and $D$ in equation (2.16)), we obtain [32]

$$
\begin{aligned}
& F_{\mu \nu \rho}^{(q: d)}+\left.F_{\mu \nu \rho}^{(q: e)}\right|_{\substack{\text { relevant } \\
\text { terms }}}= \\
&=2 i g^{2} g^{(1 q)} f^{a b c} \int_{0}^{1} d x \int_{0}^{1-x} d y \int \frac{d^{4} l}{(2 \pi)^{4}} \frac{p_{\nu} q_{\mu} x y\left[p_{\rho}(1-2 x)+q_{\rho}(2 y-1)\right]}{\left[l^{2}+2 x y p \cdot q-m_{q}^{2}\right]^{3}} \pm \\
& \quad \pm 2 i g^{2} g^{(1 q)} d^{a b c} \int_{0}^{1} d x \int_{0}^{1-x} d y \int \frac{d^{4} l}{(2 \pi)^{4}} \frac{x y\left(\epsilon_{\mu \rho \gamma \delta} p^{\gamma} q^{\delta} p_{\nu}-\epsilon_{\nu \rho \gamma \delta} p^{\gamma} q^{\delta} q_{\mu}\right)}{\left[l^{2}+2 x y p \cdot q-m_{q}^{2}\right]^{3}}
\end{aligned}
$$

The momentum integrals may be evaluated by standard techniques to obtain

$$
\begin{gathered}
F_{\mu \nu \rho}^{(q: d)}+\left.F_{\mu \nu \rho}^{(q: e)}\right|_{\substack{\text { relevant } \\
\text { terms }}}= \\
=\frac{g^{2} g^{(1 q)} f^{a b c}}{(4 \pi)^{2}} \int_{0}^{1} d x \int_{0}^{1-x} d y \frac{p_{\nu} q_{\mu} x y\left[p_{\rho}(1-2 x)+q_{\rho}(2 y-1)\right]}{m_{q}^{2}-2 x y p \cdot q} \pm \\
\pm \frac{g^{2} g^{(1 q)} d^{a b c}}{(4 \pi)^{2}}\left(\epsilon_{\mu \rho \gamma \delta} p^{\gamma} q^{\delta} p_{\nu}-\epsilon_{\nu \rho \gamma \delta} p^{\gamma} q^{\delta} q_{\mu}\right) \int_{0}^{1} d x \int_{0}^{1-x} d y \frac{x y}{m_{q}^{2}-2 x y p \cdot q}= \\
=\frac{2 g^{2} g^{(1 q)} f^{a b c}}{(4 \pi)^{2}} q_{\mu} p_{\nu} p_{\rho} I\left(m_{q}, M_{\mathrm{KK}}\right) \pm \frac{g^{2} g^{(1 q)} d^{a b c}}{(4 \pi)^{2}}\left(\epsilon_{\mu \rho \gamma \delta} p^{\gamma} q^{\delta} p_{\nu}-\epsilon_{\nu \rho \gamma \delta} p^{\gamma} q^{\delta} q_{\mu}\right) K\left(m_{q}, M_{\mathrm{KK}}\right),
\end{gathered}
$$

where

$$
I\left(m_{q}, M_{\mathrm{KK}}\right)=\int_{0}^{1} d x \int_{0}^{1-x} d y \frac{x y(1-x-y)}{m_{q}^{2}-x y M_{\mathrm{KK}}^{2}}
$$


and

$$
K\left(m_{q}, M_{\mathrm{KK}}\right)=\int_{0}^{1} d x \int_{0}^{1-x} d y \frac{x y}{m_{q}^{2}-x y M_{\mathrm{KK}}^{2}} .
$$

So each quark loop contributes a total of $-\frac{2 g^{2} g^{(1 q)} f^{a b c}}{(4 \pi)^{2}} I\left(m_{q}, M_{\mathrm{KK}}\right)$ to the coefficient $A$ in equation (2.16), a total of $\mp \frac{g^{2} g^{(1 q)} d^{a b c}}{(4 \pi)^{2}} K\left(m_{q}, M_{\mathrm{KK}}\right)$ to the coefficient $C$ and a total of $\pm \frac{g^{2} g^{(1 q)} d^{a b c}}{(4 \pi)^{2}} K\left(m_{q}, M_{\mathrm{KK}}\right)$ to the coefficient $D$, where the sign of the contribution varies as the quark is right- or left-handed. (We shall evaluate the integral $I\left(m_{q}, M_{\mathrm{KK}}\right)$ later; it will turn out that we shall not need the integral $K\left(m_{q}, M_{\mathrm{KK}}\right)$.)

\subsection{Contribution from diagrams with Kaluza-Klein gluon (or ghost) loops}

We have established that we only need to consider diagram (j) in the KK gluon loop sector and diagram (d) from the KK ghost loop. It is useful first to derive an identity for the $\mathrm{SU}(3)$ structure constants contained in the expressions for these diagrams.

The structure constants $f^{a b c}$ satisfy the identity

$$
\left(T^{a}\right)_{b c}=-i f^{a b c},
$$

where the $T^{a}$ are in the adjoint representation of $\mathrm{SU}(3)$ and satisfy the same algebra as the fundamental representation. It therefore follows that

$$
\begin{aligned}
f^{a d h} f^{c f d} f^{b h f} & =-i\left(T^{a}\right)_{d h}\left(T^{c}\right)_{f d}\left(T^{b}\right)_{h f}= \\
& =-i \operatorname{Tr}\left(T^{a} T^{b} T^{c}\right)= \\
& =\frac{1}{4}\left(f^{a b c}-i d^{a b c}\right)
\end{aligned}
$$

using equation (5.2).

We may now apply a Feynman parametrisation and integral redefinition to equations (4.15) and (4.19) and evaluate the numerators [32], obtaining

$$
\begin{aligned}
F_{\mu \nu \rho}^{(g: j)} & =-\frac{9}{2} g^{2} g^{(111)}\left(i f^{a b c}+d^{a b c}\right) q_{\mu} p_{\nu} p_{\rho} \int_{0}^{1} d x \int_{0}^{1-x} d y \int \frac{d^{4} l}{(2 \pi)^{4}} \frac{x y(1-x-y)}{\left[l^{2}+2 x y p \cdot q-M_{\mathrm{KK}}^{2}\right]^{3}}, \\
F_{\mu \nu \rho}^{(\mathrm{gh}: d)} & =\frac{1}{4} g^{2} g^{(111)}\left(i f^{a b c}+d^{a b c}\right) q_{\mu} p_{\nu} p_{\rho} \int_{0}^{1} d x \int_{0}^{1-x} d y \int \frac{d^{4} l}{(2 \pi)^{4}} \frac{x y(1-x-y)}{\left[l^{2}+2 x y p \cdot q-M_{\mathrm{KK}}^{2}\right]^{3}} .
\end{aligned}
$$

The momentum integrals may be evaluated by standard techniques to obtain

$$
\begin{aligned}
F_{\mu \nu \rho}^{(g: j)} & =-\frac{9}{4} \frac{g^{2} g^{(111)}}{(4 \pi)^{2}}\left(f^{a b c}-i d^{a b c}\right) q_{\mu} p_{\nu} p_{\rho} I\left(M_{\mathrm{KK}}, M_{\mathrm{KK}}\right), \\
F_{\mu \nu \rho}^{(\mathrm{gh}: d)} & =\frac{1}{8} \frac{g^{2} g^{(111)}}{(4 \pi)^{2}}\left(f^{a b c}-i d^{a b c}\right) q_{\mu} p_{\nu} p_{\rho} I\left(M_{\mathrm{KK}}, M_{\mathrm{KK}}\right),
\end{aligned}
$$

where $I\left(M_{\mathrm{KK}}, M_{\mathrm{KK}}\right)$ is defined in equation (5.9).

We therefore obtain a contribution of $\frac{17}{8} \frac{g^{2} g^{(111)}}{(4 \pi)^{2}}\left(f^{a b c}-i d^{a b c}\right) I\left(M_{\mathrm{KK}}, M_{\mathrm{KK}}\right)$ to the coefficient $A$ in equation (2.16) from the KK gluon and KK ghost loops. 


\subsection{Overall amplitude before anomaly cancellation}

Summing the contributions from the quark loop diagrams and the KK gluon and KK ghost loop diagrams, we derive that the amplitude for the process $g g \rightarrow g_{\mathrm{KK}}$, neglecting higher order excitations of the KK gluon and KK ghost, satisfies

$$
\begin{aligned}
F_{\mu \nu \rho}=A\left(\eta_{\mu \nu} p \cdot q\right. & \left.-q_{\mu} p_{\nu}\right) p_{\rho}+ \\
& +C\left(\epsilon_{\mu \nu \rho \gamma} p^{\gamma} p \cdot q-\epsilon_{\mu \nu \rho \gamma} q^{\gamma} p \cdot q-\epsilon_{\mu \rho \gamma \delta} p^{\gamma} q^{\delta} p_{\nu}+\epsilon_{\nu \rho \gamma \delta} p^{\gamma} q^{\delta} q_{\mu}\right),
\end{aligned}
$$

where

$$
\begin{aligned}
& A=\frac{17}{8} \frac{g^{2} g^{(111)}}{(4 \pi)^{2}}\left(f^{a b c}-i d^{a b c}\right) I\left(M_{\mathrm{KK}}, M_{\mathrm{KK}}\right)-\sum_{q_{L}, q_{R}} \frac{2 g^{2} g^{(1 q)} f^{a b c}}{(4 \pi)^{2}} I\left(m_{q}, M_{\mathrm{KK}}\right), \\
& C=\sum_{q_{R}} \frac{g^{2} g^{(1 q)} d^{a b c}}{(4 \pi)^{2}} K\left(m_{q}, M_{\mathrm{KK}}\right)-\sum_{q_{L}} \frac{g^{2} g^{(1 q)} d^{a b c}}{(4 \pi)^{2}} K\left(m_{q}, M_{\mathrm{KK}}\right)
\end{aligned}
$$

the expressions for the integrals $I$ and $K$ are given in equations (5.9) and (5.10), respectively, and we emphasise that the left- and right-handed quark states must be treated as separate particles in the sum for $A$.

At this stage, we note that the amplitude as calculated so far contains an anomaly in the current associated with the outgoing KK gluon (that is, the on-shell Ward identity $r^{\rho} F_{\mu \nu \rho}=0$ is not satisfied). This is because we have taken the gauge $A_{4}=0$, which we do not have the freedom to do in a five-dimensional non-Abelian theory with chiral delocalised quarks if we desire anomaly cancellation [29]. We must therefore now apply a gauge transformation that leaves the four-dimensional theory anomaly-free. We note that from the perspective of our current calculation, this is a technical requirement that does not affect the final result of the on-shell calculation. However, it does have the potential to affect the result for $F_{\mu \nu \rho}$.

\subsection{Cancellation of the anomaly}

It is possible to add to the five-dimensional Lagrangian for this theory the Chern-Simons term [33]

$$
\mathcal{L}_{\mathrm{CS}}=c \epsilon^{V W X Y Z} \operatorname{Tr}\left(A_{V} \partial_{W} A_{X} \partial_{Y} A_{Z}-\frac{3 i}{2} A_{V} A_{W} A_{X} \partial_{Y} A_{Z}-\frac{3}{5} A_{V} A_{W} A_{X} A_{Y} A_{Z}\right),
$$

where $A_{V}=A_{V}^{a} t^{a}$, etc and $V, W, X, \ldots$ are 5 -dimensional space-time indices. Of interest to us are the three-point $g g_{\mathrm{KK}}$ interaction vertices that result from this Lagrangian term (the other vertices will only feature in higher-order corrections). Without making a specific gauge choice, but keeping the form of the extra-dimensional wavefunctions noted in section 6.3 (which we shall justify momentarily), we obtain from the four-dimensional perspective two types of interaction term, depending upon whether the index for the extra dimension attaches to a gauge field or to a derivative. The terms are the four-dimensional Chern-Simons-like term

$$
\mathcal{L}_{\mathrm{CS} 4}=c_{1} \epsilon^{\mu \nu \rho \gamma} d^{a b c} A_{\mu}^{(0) a} \partial_{\gamma} A_{\nu}^{(0) b} \partial_{4} A_{\rho}^{(1) c}
$$




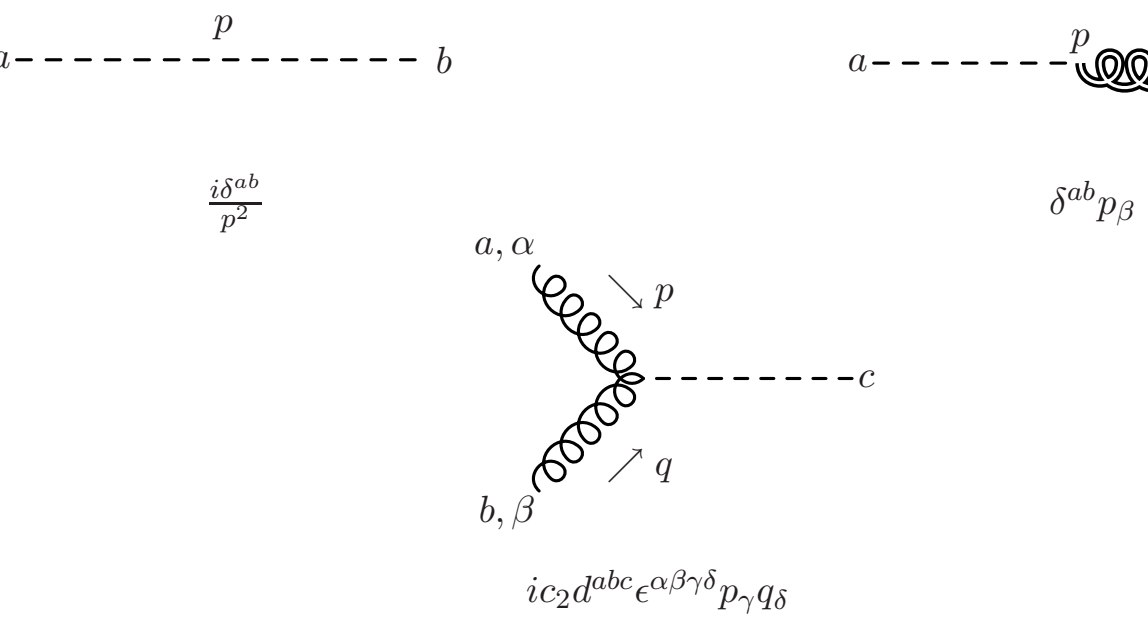

Figure 5. Additional relevant non-zero Feynman rules for gauges with $A_{4} \neq 0$.

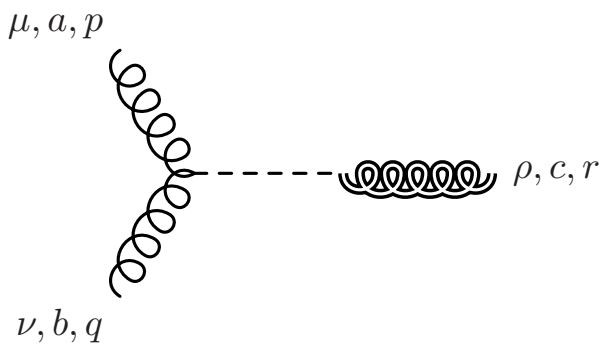

Figure 6. Additional Feynman diagram for the process $g g \rightarrow g_{\mathrm{KK}}$ for gauges with $A_{4} \neq 0$.

(we note that the $\partial_{4}$ can only act on the KK mode as the extra-dimensional wavefunctions of the zero modes are flat, and all other possible terms can be obtained by using symmetry or antisymmetry arguments from the one given), and the three-point "axion" term

$$
\mathcal{L}_{\mathrm{Ax}}=c_{2} \epsilon^{\mu \nu \gamma \delta} d^{a b c}\left(\partial_{\gamma} A_{\mu}^{a} \partial_{\delta} A_{\nu}^{b} A_{4}^{c}+2 A_{\mu}^{a} \partial_{\gamma} A_{\nu}^{b} \partial_{\delta} A_{4}^{c}\right) .
$$

The constants $c_{1}$ and $c_{2}$ may be chosen separately, since we inherit two degrees of freedom from the five-dimensional theory, one from the coefficient of the five-dimensional ChernSimons term, and one from the gauge choice. We choose $c_{1}=0$, and shall choose $c_{2}$ so as to provide the requisite anomaly cancellation.

The $A_{4}$ field also enters into the kinetic gauge term of the Lagrangian, allowing oscillation between the scalar axion and the four-dimensional gauge modes.

The additional Feynman rules resulting from the change of gauge and the addition of the extra Lagrangian term are shown in figure 5.

These rules give rise to one extra diagram, shown in figure 6 . 
The extra diagram gives a contribution to the amplitude of

$$
F_{\mu \nu \rho}^{\text {(axion) }}=-c_{2} d^{a b c} \frac{1}{r^{2}} \epsilon_{\mu \nu \gamma \delta} p^{\gamma} q^{\delta} r_{\rho},
$$

and we can choose the constant $c_{2}$ so that this contribution is equal on-shell to

$$
C \epsilon_{\mu \nu \gamma \delta} p^{\gamma} q^{\delta} r_{\rho}
$$

where $C$ is as given in equation (5.19). This addition means that the amplitude now satisfies the Ward identity $r^{\rho} F_{\mu \nu \rho}=0$, without disturbing the other Ward identities ${ }^{2}$. Since the term $r_{\rho}$ in the additional contribution is projected out by the KK polarisation tensor, it is clear that this contribution makes no difference to the square of the final on-shell matrix element. (We recall that the on-shell $g_{\mathrm{KK}}$ amplitude is given by contracting $F_{\mu \nu \rho}$ with the external polarisation vectors.)

Before finishing this technical aside, we note that making a gauge choice other than $A_{4}=0$ alters the form of the five-dimensional Yang-Mills equation [11, 28], and in particular this has the potential to break the derivation of the extra-dimensional wavefunction $\chi^{(0)}$ as constant. However, this alteration may be viewed as a loop-level correction to the equation, so that the effects of this alteration upon all the diagrams we have considered so far are twoloop level. This alteration does have the potential to produce a tree diagram for $g g \rightarrow g_{\mathrm{KK}}$ fusion, but the structure of such a diagram is such that it may be absorbed into the threepoint counterterm diagram and neglected from the perspective of a separate contribution.

\section{Calculation of the production cross-section}

Our first step in obtaining the production cross-section is to obtain the square of the matrix element. For this we may use the polarisation sum formulae

$$
\begin{aligned}
\varepsilon^{\mu}(p) \varepsilon^{\mu^{\prime} *}(p) & =-\eta^{\mu \mu^{\prime}}+Q^{\mu \mu^{\prime}}, \\
\varepsilon^{\nu}(q) \varepsilon^{\nu^{\prime} *}(q) & =-\eta^{\nu \nu^{\prime}}+Q^{\nu \nu^{\prime}}, \\
\varepsilon_{g_{\mathrm{KK}}}^{\rho^{\prime}}(r) \varepsilon_{g_{\mathrm{KK}}}^{\rho *}(r) & =\frac{r^{\rho} r^{\rho^{\prime}}}{M_{\mathrm{KK}}^{2}}-\eta^{\rho \rho^{\prime}},
\end{aligned}
$$

where $Q^{\mu \mu^{\prime}}=\left(p^{\mu} q^{\mu^{\prime}}+p^{\mu^{\prime}} q^{\mu}\right) /(p \cdot q)$ and $Q^{\nu \nu^{\prime}}=\left(q^{\nu} p^{\nu^{\prime}}+q^{\nu^{\prime}} p^{\nu}\right) /(p \cdot q)$. The square of the matrix element satisfies

$$
|\mathcal{M}|^{2}=\varepsilon^{\mu}(p) \varepsilon^{\nu}(q) \varepsilon_{g_{\mathrm{KK}}}^{\rho *}(r) F_{\mu \nu \rho} \varepsilon^{\mu^{\prime} *}(p) \varepsilon^{\nu^{\prime} *}(q) \varepsilon_{g_{\mathrm{KK}}}^{\rho^{\prime}}(r) F_{\mu^{\prime} \nu^{\prime} \rho^{\prime}}^{*},
$$

and substituting for the polarisation sum formulae and using equation (5.17), we obtain [32]

$$
|\mathcal{M}|^{2}=\frac{M_{\mathrm{KK}}^{6}}{32}|A|^{2},
$$

\footnotetext{
${ }^{2}$ To prove the Ward identity fully it is also necessary to note that the term $A\left(\eta_{\mu \nu} p \cdot q-q_{\mu} p_{\nu}\right) p_{\rho}$ was derived by applying the KK polarisation tensor to the term $(A / 2)\left(\eta_{\mu \nu} p \cdot q-q_{\mu} p_{\nu}\right)\left(p_{\rho}-q_{\rho}\right)$.
} 
with $A$ as given in equation (5.18), and where we have averaged over incoming polarisation states. We note that the coefficient $C$ has cancelled and we therefore do not need to evaluate the integrals $K$ that feature in the equation for coefficient $C$ and not in the equation for coefficient $A$.

We may evaluate $|A|^{2}$, incorporating averaging over incoming colour states and summing over outgoing colour states to obtain

$$
\begin{aligned}
|\mathcal{M}|^{2}=\frac{M_{\mathrm{KK}}^{6} g^{4}}{(4 \pi)^{4}} \mid & \frac{4046}{16384} g^{(111)^{2}}\left[I\left(M_{\mathrm{KK}}, M_{\mathrm{KK}}\right)\right]^{2}- \\
& -\frac{51}{256} g^{(111)} \sum_{q_{L}, q_{R}} g^{(1 q)}\left[I\left(M_{\mathrm{KK}}, M_{\mathrm{KK}}\right) I\left(m_{q}, M_{\mathrm{KK}}\right)\right]+ \\
& +\frac{3}{64}\left[\sum_{q_{L}, q_{R}} g^{(1 q)} I\left(m_{q}, M_{\mathrm{KK}}\right)\right]^{2} \mid
\end{aligned}
$$

where we have combined the expressions resulting from the real and imaginary parts of the matrix element to obtain this last expression.

Writing

$$
|\mathcal{M}|^{2}=\frac{M_{\mathrm{KK}}^{6} g^{4}}{(4 \pi)^{4}}|\tilde{\mathcal{M}}|^{2},
$$

we can write an expression for the cross-section for the production of on-shell KK gluons from the gluon-initiated states as

$$
\sigma=\frac{M_{\mathrm{KK}}^{2} \alpha_{s}^{2}}{8 \pi} \int d y x_{1} g_{a}\left(x_{1}, M_{\mathrm{KK}}^{2}\right) x_{2} g_{b}\left(x_{2}, M_{\mathrm{KK}}^{2}\right)|\tilde{\mathcal{M}}|^{2},
$$

where $x_{1,2}=\sqrt{\tau} e^{ \pm y}$, with $\sqrt{\tau}=M_{\mathrm{KK}} / \sqrt{s}, y$ being the rapidity of the KK gluon and $\sqrt{s}$ being the total centre of mass energy of the $p p$ system.

We have used this expression to calculate the cross-section for the KK gluon from the $g g$-initial state and compared it with the leading order $q \bar{q}$ result (using the LO crosssection presented in ref. [22]) at the Large Hadron Collider (LHC), assuming a centre-ofmass energy of $14 \mathrm{TeV}$. The ratio is plotted for some typical values of the KK gluon mass in figure 7. The cross-section from the $g g$ NLO subprocesses turns out to be less than a thousandth of the LO cross section. This is, in turn, due to appearance of the large KK gluon mass squared in the denominators of the integral $I$ which have been analytically studied in appendix B to provide some intuitive basis for these numerical results.

In principle, to complete the full calculation of the KK gluon cross-section at NLO one needs to calculate the $q \bar{q}$-initiated diagrams at NLO. But given that the $g g$-initiated contribution is tiny, it is expected that the $q \bar{q}$-initiated contribution will be even smaller due to the suppressed couplings of valence quarks and the calculation is, therefore, not of much interest.

\section{Discussion}

As expected, the most significant contribution to the $g g \rightarrow g_{\mathrm{KK}}$ production process comes from the $t_{R}$ loop, which has the strongest coupling to the $g_{\mathrm{KK}}$ and interferes constructively 


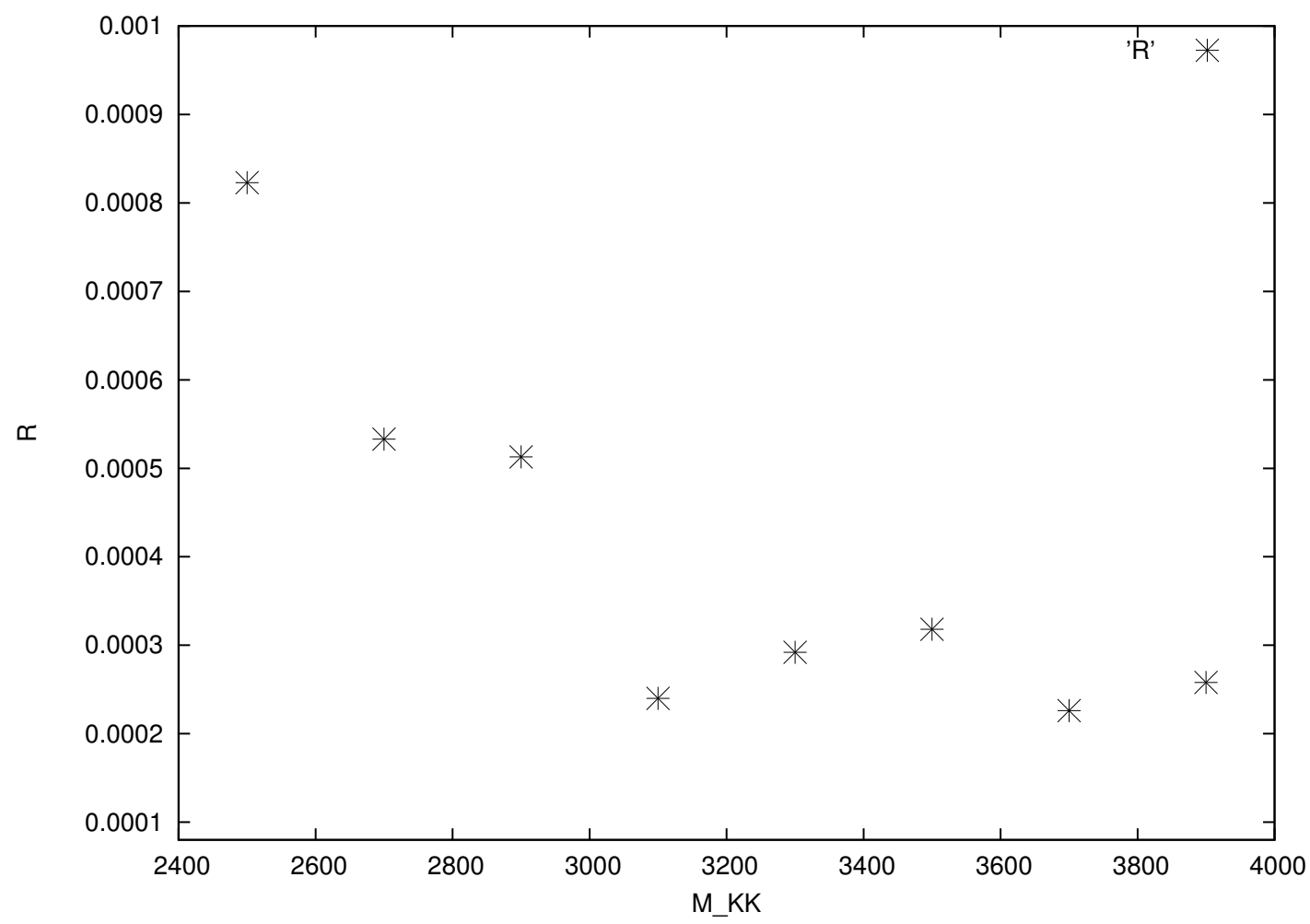

Figure 7. The ratio of the gluon-initiated NLO and the $q \bar{q}$-initiated LO cross-sections for the production of a KK gluon at the LHC with $14 \mathrm{TeV}$ centre of mass energy.

with other loop particles. Other loops not involving $t_{R}$ contribute non-negligibly to the process however, owing partly to the number of different additional loops. We note that our final result disagrees with a preliminary result obtained as part of ref. [24]. Like ref. [24], we also find that the gg-initiated contribution is negligibly small.

It has been suggested [24] that the decay width for $g_{\mathrm{KK}} \rightarrow t \bar{t} / b \bar{b}$ is sufficiently large to suggest that the narrow width approximation would be an inaccurate approximation to the total matrix element. In this case, off-shell $g_{\mathrm{KK}}$ effects would be non-negligible and one would like to generalise our calculation to the off-shell case. In appendix C, we have provided a recipe to modify the amplitudes presented here to the case where the $g_{\mathrm{KK}}$ is off-shell. However, we should remember that this alone is not enough for a full study of the off-shell effects for such a study would have to include the interference effects with the Standard Model. The full calculation of the interference effects is, however, not of much interest given the diminutiveness of the effects in the on-shell case.

\section{Acknowledgments}

This work has been partially supported by STFC. We thank G. Moreau for communication about the calculation. BCA and JPS would like to thank Howard Haber, Bryan Webber and the members of the Cambridge Supersymmetry Working Group for useful discussions. Part of this work was carried out at the 'Les Houches 2009: Physics at TeV Colliders' and 
'From Strings to LHC II' workshops. KS wishes to thank IPPP, Durham and LAPTH, Annecy for visits during which this work was completed.

\section{A Tensors satisfying the general form of section 2}

The following combinations of $p, q$, the metric tensor $\eta$, and the Levi-Civita tensor $\epsilon$, satisfy the conditions given in section 2 that must be satisfied by the tensor $t_{\mu \nu \rho \alpha \beta}^{1}$, as defined by equation (2.10). Degenerate combinations have been removed (so, for example, terms with a $q_{\rho}$ are replaced by terms with $-p_{\rho}$, and terms with more than one $\epsilon$ tensor are removed in favour of metric tensors). Each term may have a scalar coefficient.

$$
\begin{gathered}
\eta_{\alpha \beta} p_{\nu} q_{\mu} p_{\rho}-\eta_{\mu \beta} p_{\nu} q_{\alpha} p_{\rho}-\eta_{\alpha \nu} p_{\beta} q_{\mu} p_{\rho}+\eta_{\mu \nu} p_{\beta} q_{\alpha} p_{\rho} \\
\eta_{\alpha \beta} \eta_{\mu \nu} p_{\rho}-\eta_{\mu \beta} \eta_{\alpha \nu} p_{\rho} \\
\eta_{\alpha \beta} \eta_{\mu \rho} p_{\nu}-\eta_{\mu \beta} \eta_{\alpha \rho} p_{\nu}-\eta_{\alpha \nu} \eta_{\mu \rho} p_{\beta}+\eta_{\mu \nu} \eta_{\alpha \rho} p_{\beta} \\
\eta_{\beta \alpha} \eta_{\nu \rho} q_{\mu}-\eta_{\nu \alpha} \eta_{\beta \rho} q_{\mu}-\eta_{\beta \mu} \eta_{\nu \rho} q_{\alpha}+\eta_{\nu \mu} \eta_{\beta \rho} q_{\alpha} \\
\epsilon_{\mu \nu \alpha \beta} p_{\rho} \\
\epsilon_{\mu \nu \rho \alpha} p_{\beta}-\epsilon_{\mu \beta \rho \alpha} p_{\nu} \\
\epsilon_{\mu \nu \rho \beta} q_{\alpha}-\epsilon_{\alpha \nu \rho \beta} q_{\mu} \\
\epsilon_{\mu \nu \rho \gamma} p^{\gamma} p_{\beta} q_{\alpha}-\epsilon_{\alpha \nu \rho \gamma} p^{\gamma} p_{\beta} q_{\mu}-\epsilon_{\mu \beta \rho \gamma} p^{\gamma} p_{\nu} q_{\alpha}+\epsilon_{\alpha \beta \rho \gamma} p^{\gamma} p_{\nu} q_{\mu} \\
\epsilon_{\mu \nu \rho \gamma} q^{\gamma} p_{\beta} q_{\alpha}-\epsilon_{\alpha \nu \rho \gamma} q^{\gamma} p_{\beta} q_{\mu}-\epsilon_{\mu \beta \rho \gamma} q^{\gamma} p_{\nu} q_{\alpha}+\epsilon_{\alpha \beta \rho \gamma} q^{\gamma} p_{\nu} q_{\mu} \\
\epsilon_{\mu \nu \rho \gamma} p^{\gamma} \eta_{\alpha \beta}-\epsilon_{\alpha \nu \rho \gamma} p^{\gamma} \eta_{\mu \beta}-\epsilon_{\mu \beta \rho \gamma} p^{\gamma} \eta_{\nu \alpha}+\epsilon_{\alpha \beta \rho \gamma} p^{\gamma} \eta_{\nu \mu} \\
\epsilon_{\mu \nu \rho \gamma} q^{\gamma} \eta_{\alpha \beta}-\epsilon_{\alpha \nu \rho \gamma} q^{\gamma} \eta_{\mu \beta}-\epsilon_{\mu \beta \rho \gamma} q^{\gamma} \eta_{\nu \alpha}+\epsilon_{\alpha \beta \rho \gamma} q^{\gamma} \eta_{\nu \mu} \\
\epsilon_{\mu \nu \alpha \gamma} p^{\gamma} p_{\rho} p_{\beta}-\epsilon_{\mu \beta \alpha \gamma} p^{\gamma} p_{\rho} p_{\nu} \\
\epsilon_{\mu \nu \alpha \gamma} q^{\gamma} p_{\rho} p_{\beta}-\epsilon_{\mu \beta \alpha \gamma} q^{\gamma} p_{\rho} p_{\nu} \\
\epsilon_{\mu \nu \alpha \gamma} p^{\gamma} \eta_{\rho \beta}-\epsilon_{\mu \beta \alpha \gamma} p^{\gamma} \eta_{\rho \nu} \\
\epsilon_{\mu \nu \alpha \gamma} q^{\gamma} \eta_{\rho \beta}-\epsilon_{\mu \beta \alpha \gamma} q^{\gamma} \eta_{\rho \nu} \\
\epsilon_{\mu \nu \beta \gamma} p^{\gamma} p_{\rho} q_{\alpha}-\epsilon_{\alpha \nu \beta \gamma} p^{\gamma} p_{\rho} q_{\mu} \\
\epsilon_{\mu \nu \beta \gamma} q^{\gamma} p_{\rho} q_{\alpha}-\epsilon_{\alpha \nu \beta \gamma} q^{\gamma} p_{\rho} q_{\mu} \\
\epsilon_{\mu \nu \beta \gamma} p^{\gamma} \eta_{\rho \alpha}-\epsilon_{\alpha \nu \beta \gamma} p^{\gamma} \eta_{\rho \mu} \\
\epsilon_{\mu \nu \beta \gamma} q^{\gamma} \eta_{\rho \alpha}-\epsilon_{\alpha \nu \beta \gamma} q^{\gamma} \eta_{\rho \mu}
\end{gathered}
$$




\section{B Analytic evaluation of the Feynman parameter integral used in the calculation}

In the calculation we arrive at the integral (equation (5.9))

$$
I\left(m_{q}, M_{\mathrm{KK}}\right)=\int_{0}^{1} d x \int_{0}^{1-x} d y \frac{x y(1-x-y)}{m_{q}^{2}-x y M_{\mathrm{KK}}^{2}} .
$$

We note that in the integration region the expression $x y$ has a maximum value of $1 / 4$, so for Standard Model quarks (where $M_{\mathrm{KK}} \gg 2 m_{q}$ ) we may approximate the integral by $I\left(0, M_{\mathrm{KK}}\right)$, which gives us

$$
I\left(m_{q}, M_{\mathrm{KK}}\right) \approx-\frac{1}{6 M_{\mathrm{KK}}^{2}} .
$$

We also obtain the integral $I\left(M_{\mathrm{KK}}, M_{\mathrm{KK}}\right)$. This may be evaluated as follows:

$$
\begin{aligned}
\int_{0}^{1} d x \int_{0}^{1-x} d y \frac{x y(1-x-y)}{m_{q}^{2}-x y M_{\mathrm{KK}}^{2}} & = \\
& =\frac{1}{M_{\mathrm{KK}}^{2}} \int_{0}^{1} d x \int_{0}^{1-x} d y(x+y-1)+\frac{1-x-y}{1-x y}= \\
& =\frac{1}{M_{\mathrm{KK}}^{2}} \int_{0}^{1} d x \int_{0}^{1-x} d y(2 x-1)+\frac{1-2 x}{1-x y}
\end{aligned}
$$

since the integration region is symmetrical about the line $x=y$ so we may interchange $x$ and $y$ in any term in the integrand. We may evaluate this to obtain

$$
\begin{aligned}
- & \frac{1}{6 M_{\mathrm{KK}}^{2}}+\frac{1}{M_{\mathrm{KK}}^{2}} \int_{0}^{1} d x\left[\frac{(2 x-1)}{x} \log (1-x y)\right]_{y=0}^{y=1-x}= \\
= & -\frac{1}{6 M_{\mathrm{KK}}^{2}}+\frac{1}{M_{\mathrm{KK}}^{2}} \int_{0}^{1} d x\left(2-\frac{1}{x}\right) \log \left(x^{2}-x+1\right)= \\
= & -\frac{1}{6 M_{\mathrm{KK}}^{2}}+\frac{1}{M_{\mathrm{KK}}^{2}} \int_{0}^{1} d x\left(2-\frac{1}{x}\right)\left[\log \left(x-\frac{1}{2}-i \frac{\sqrt{3}}{2}\right)+\log \left(x-\frac{1}{2}+i \frac{\sqrt{3}}{2}\right)\right]= \\
= & -\frac{1}{6 M_{\mathrm{KK}}^{2}}+\frac{2}{M_{\mathrm{KK}}^{2}}\left(-2+\frac{\pi}{\sqrt{3}}\right)- \\
& -\frac{1}{M_{\mathrm{KK}}^{2}} \int_{0}^{1} d x \frac{1}{x}\left[\log \left(1-\frac{x}{\frac{1}{2}+i \frac{\sqrt{3}}{2}}\right)+\log \left(1-\frac{x}{\frac{1}{2}+i \frac{\sqrt{3}}{2}}\right)\right]= \\
= & \frac{1}{M_{\mathrm{KK}}^{2}}\left(\frac{2 \pi}{\sqrt{3}}-\frac{25}{6}\right)+\frac{1}{M_{\mathrm{KK}}^{2}}\left[\operatorname{Li}_{2}\left(\frac{1}{\frac{1}{2}+i \frac{\sqrt{3}}{2}}\right)+\mathrm{Li}_{2}\left(\frac{1}{\frac{1}{2}-i \frac{\sqrt{3}}{2}}\right)\right]= \\
= & \frac{1}{M_{\mathrm{KK}}^{2}}\left(\frac{2 \pi}{\sqrt{3}}-\frac{25}{6}\right)+\frac{1}{M_{\mathrm{KK}}^{2}}\left[\operatorname{Li}_{2}\left(\frac{1}{2}-i \frac{\sqrt{3}}{2}\right)+\mathrm{Li}_{2}\left(\frac{1}{2}+i \frac{\sqrt{3}}{2}\right)\right]= \\
= & \frac{1}{M_{\mathrm{KK}}^{2}}\left(\frac{2 \pi}{\sqrt{3}}-\frac{25}{6}\right)+\frac{1}{M_{\mathrm{KK}}^{2}}\left[\frac{\pi^{2}}{6}-\log \left(\frac{1}{2}-i \frac{\sqrt{3}}{2}\right) \log \left(\frac{1}{2}+i \frac{\sqrt{3}}{2}\right)\right]=
\end{aligned}
$$




$$
\begin{aligned}
& =\frac{1}{M_{\mathrm{KK}}^{2}}\left(\frac{2 \pi}{\sqrt{3}}-\frac{25}{6}\right)+\frac{1}{M_{\mathrm{KK}}^{2}}\left[\frac{\pi^{2}}{6}-\frac{\pi^{2}}{9}\right]= \\
& =\frac{1}{M_{\mathrm{KK}}^{2}}\left(\frac{\pi^{2}}{18}+\frac{2 \pi}{\sqrt{3}}-\frac{25}{6}\right) \approx \frac{1}{100 M_{\mathrm{KK}}^{2}},
\end{aligned}
$$

where we have used a number of standard properties of the dilogarithm function (see, e.g. appendix E.2 of reference [34]).

A similar approach may be used to obtain a full analytic solution to the integral $I\left(m_{q}, M_{\mathrm{KK}}\right)$, although because the dilogarithms do not simplify as usefully in that case the utility of the overall solution is comparatively smaller.

\section{Generalisation of our results to off-shell KK gluon production}

We note that the argument of section 2 only assumes that the KK gluon is on-shell in allowing the replacement $p_{\rho} \leftrightarrow-q_{\rho}$, so the argument still holds with the exception that we obtain additional possible terms in the general form of the amplitude, which correspond to replacing $p_{\rho}$ with $q_{\rho}$ in equations (2.12) and (2.13). The symmetry of the diagrams means we know that the terms we shall obtain will replace $p_{\rho}$ with $\left(p_{\rho}-q_{\rho}\right) / 2$, although when calculating the diagrams we also have to avoid making the replacements $2 p \cdot q=r^{2}=M_{\mathrm{KK}}^{2}$. In addition, because we cannot use the polarisation tensor $\varepsilon_{g_{\mathrm{KK}}}^{\rho}(r)$ when deriving the general form of the amplitude, we would have to include the term derived in equation (5.24).

Open Access. This article is distributed under the terms of the Creative Commons Attribution Noncommercial License which permits any noncommercial use, distribution, and reproduction in any medium, provided the original author(s) and source are credited.

\section{References}

[1] L. Randall and R. Sundrum, A large mass hierarchy from a small extra dimension, Phys. Rev. Lett. 83 (1999) 3370 [hep-ph/9905221] [SPIRES].

[2] W.D. Goldberger and M.B. Wise, Modulus stabilization with bulk fields, Phys. Rev. Lett. 83 (1999) 4922 [hep-ph/9907447] [SPIRES].

[3] W.D. Goldberger and M.B. Wise, Phenomenology of a stabilized modulus, Phys. Lett. B 475 (2000) 275 [hep-ph/9911457] [SPIRES].

[4] H. Davoudiasl, J.L. Hewett and T.G. Rizzo, Experimental probes of localized gravity: on and off the wall, Phys. Rev. D 63 (2001) 075004 [hep-ph/0006041] [SPIRES].

[5] K. Sridhar, Constraining the Randall-Sundrum model using diphoton production at hadron colliders, JHEP 05 (2001) 066 [hep-ph/0103055] [SPIRES].

[6] B.C. Allanach, K. Odagiri, M.A. Parker and B.R. Webber, Searching for narrow graviton resonances with the ATLAS detector at the Large Hadron Collider, JHEP 09 (2000) 019 [hep-ph/0006114] [SPIRES].

[7] B.C. Allanach et al., Exploring small extra dimensions at the large hadron collider, JHEP 12 (2002) 039 [hep-ph/0211205] [SPIRES]. 
[8] J.M. Maldacena, The large- $N$ limit of superconformal field theories and supergravity, Adv. Theor. Math. Phys. 2 (1998) 231 [Int. J. Theor. Phys. 38 (1999) 1113] [hep-th/9711200] [SPIRES].

[9] N. Arkani-Hamed, M. Porrati and L. Randall, Holography and phenomenology, JHEP 08 (2001) 017 [hep-th/0012148] [SPIRES].

[10] R. Rattazzi and A. Zaffaroni, Comments on the holographic picture of the Randall-Sundrum model, JHEP 04 (2001) 021 [hep-th/0012248] [SPIRES].

[11] A. Pomarol, Gauge bosons in a five-dimensional theory with localized gravity, Phys. Lett. B 486 (2000) 153 [hep-ph/9911294] [SPIRES].

[12] T. Gherghetta and A. Pomarol, Bulk fields and supersymmetry in a slice of AdS, Nucl. Phys. B 586 (2000) 141 [hep-ph/0003129] [SPIRES].

[13] A. Djouadi, G. Moreau and F. Richard, Resolving the $A_{\mathrm{FB}}^{b}$ puzzle in an extra dimensional model with an extended gauge structure, Nucl. Phys. B 773 (2007) 43 [hep-ph/0610173] [SPIRES].

[14] K. Agashe, A. Delgado, M.J. May and R. Sundrum, RS1, custodial isospin and precision tests, JHEP 08 (2003) 050 [hep-ph/0308036] [SPIRES].

[15] K. Agashe, A. Delgado and R. Sundrum, Gauge coupling renormalization in RS1, Nucl. Phys. B 643 (2002) 172 [hep-ph/0206099] [SPIRES].

[16] R. Contino and A. Pomarol, Holography for fermions, JHEP 11 (2004) 058 [hep-th/0406257] [SPIRES].

[17] K. Agashe, R. Contino and A. Pomarol, The minimal composite Higgs model, Nucl. Phys. B 719 (2005) 165 [hep-ph/0412089] [SPIRES].

[18] K. Agashe, G. Perez and A. Soni, B-factory signals for a warped extra dimension, Phys. Rev. Lett. 93 (2004) 201804 [hep-ph/0406101] [SPIRES].

[19] R. Contino, T. Kramer, M. Son and R. Sundrum, Warped/composite phenomenology simplified, JHEP 05 (2007) 074 [hep-ph/0612180] [SPIRES].

[20] B. Lillie, L. Randall and L.-T. Wang, The bulk RS KK-gluon at the LHC, JHEP 09 (2007) 074 [hep-ph/0701166] [SPIRES].

[21] K. Agashe, A. Belyaev, T. Krupovnickas, G. Perez and J. Virzi, LHC signals from warped extra dimensions, Phys. Rev. D 77 (2008) 015003 [hep-ph/0612015] [SPIRES].

[22] M. Guchait, F. Mahmoudi and K. Sridhar, Tevatron constraint on the Kaluza-Klein gluon of the bulk Randall-Sundrum model, JHEP 05 (2007) 103 [hep-ph/0703060] [SPIRES].

[23] M. Guchait, F. Mahmoudi and K. Sridhar, Associated production of a Kaluza-Klein

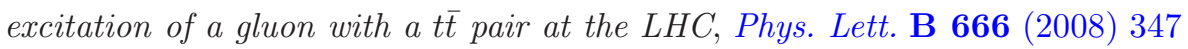
[arXiv:0710.2234] [SPIRES].

[24] A. Djouadi, G. Moreau and R.K. Singh, Kaluza-Klein excitations of gauge bosons at the LHC, Nucl. Phys. B 797 (2008) 1 [arXiv:0706.4191] [SPIRES].

[25] C.-N. Yang, Selection rules for the dematerialization of a particle into two photons, Phys. Rev. 77 (1950) 242 [SPIRES].

[26] J.F. Nieves and P.B. Pal, Gravitational decay of the Z-boson, Phys. Rev. D 72 (2005) 093006 [hep-ph/0509321] [SPIRES]. 
[27] B.C. Allanach, J.P. Skittrall and K. Sridhar, Z boson decay to photon plus Kaluza-Klein graviton in large extra dimensions, JHEP 11 (2007) 089 [arXiv:0705.1953] [SPIRES].

[28] H. Davoudiasl, J.L. Hewett and T.G. Rizzo, Bulk gauge fields in the Randall-Sundrum model, Phys. Lett. B 473 (2000) 43 [hep-ph/9911262] [SPIRES].

[29] P. Anastasopoulos, M. Bianchi, E. Dudas and E. Kiritsis, Anomalies, anomalous U(1)'s and generalized Chern-Simons terms, JHEP 11 (2006) 057 [hep-th/0605225] [SPIRES].

[30] S. Wolfram, The Mathematica book, $5^{\text {th }}$ edition, Wolfram Media Press, U.S.A. (2003).

[31] T. Hahn, CUBA: A library for multidimensional numerical integration, Comput. Phys. Commun. 168 (2005) 78 [hep-ph/0404043] [SPIRES].

[32] J.A.M. Vermaseren, New features of FORM, math-ph/0010025 [SPIRES].

[33] C.T. Hill, Anomalies, Chern-Simons terms and chiral delocalization in extra dimensions, Phys. Rev. D 73 (2006) 085001 [hep-th/0601154] [SPIRES].

[34] R.D. Field, Applications of perturbative QCD, Frontiers in physics, Addison-Wesley, U.K. (1989). 